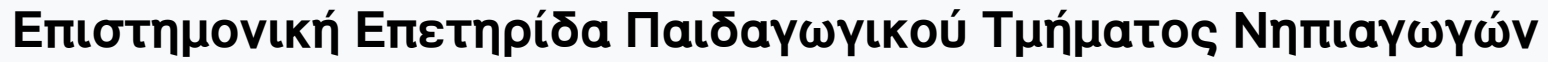 חavertotnuíou Iwavvívwv
}

Tóp. 7 (2014)

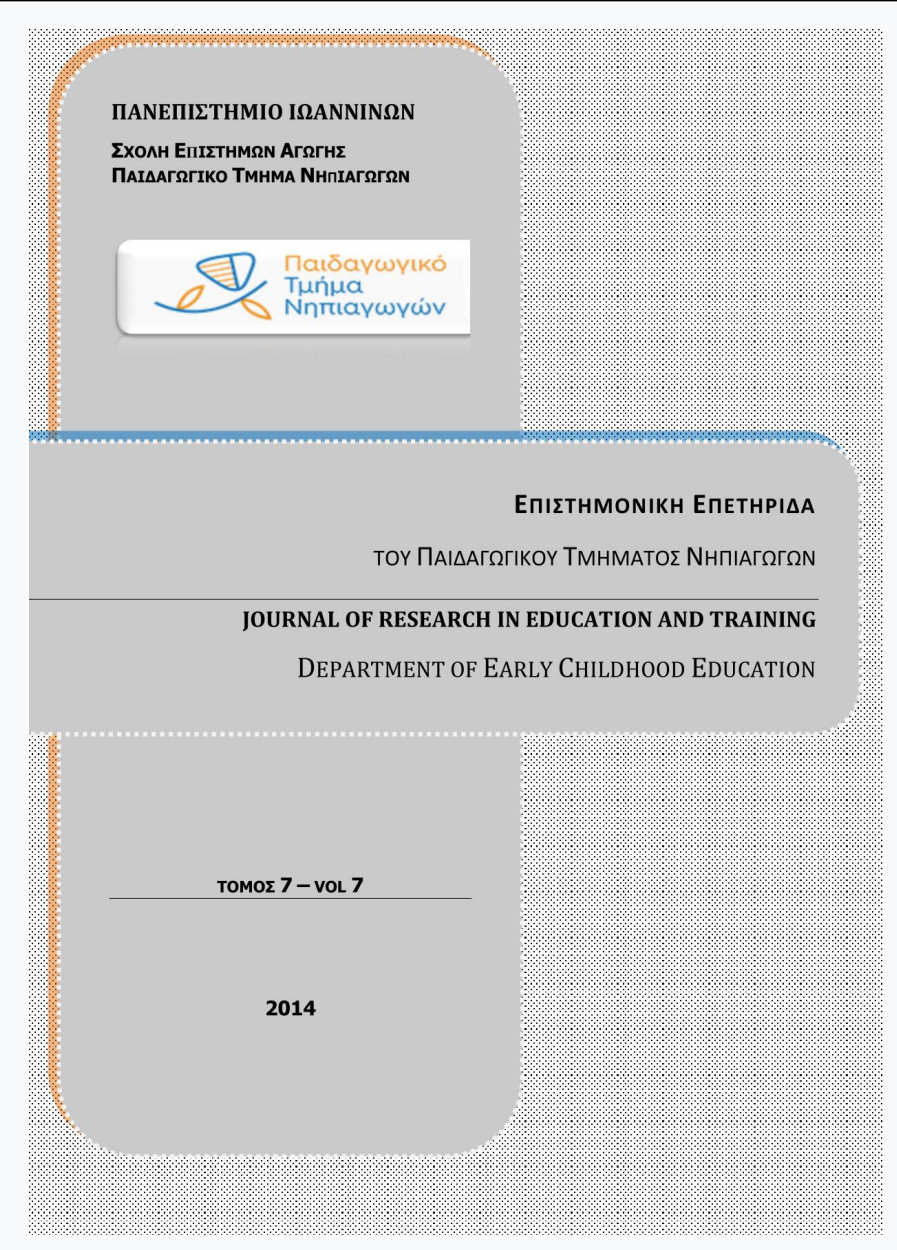

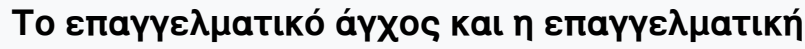

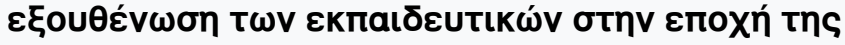
olkovoutкńs kpíons

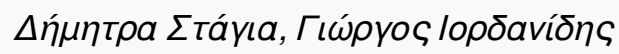

doi: $\underline{10.12681 / \text { jret.855 }}$

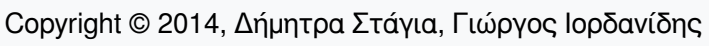

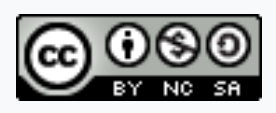

Ađ¿ıı Xpńбnৎ Creative Commons Attribution-NonCommercial-ShareAlike 4.0.

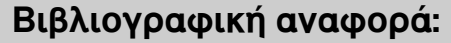

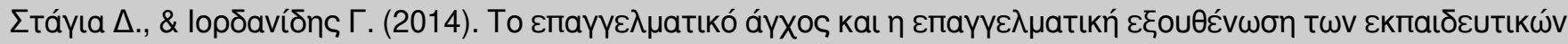

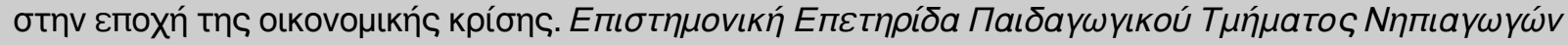

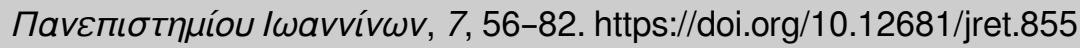




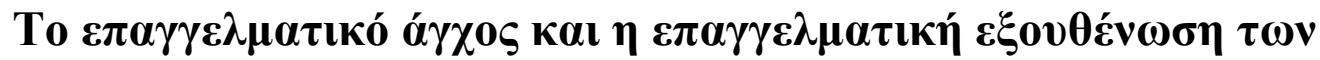

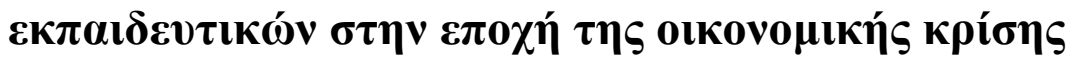

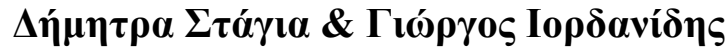

\section{ПЕРІАНЧН}

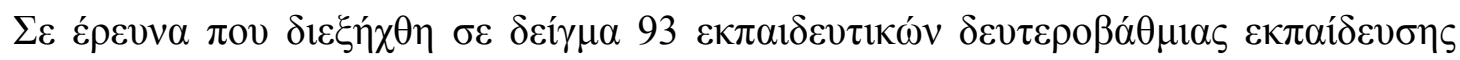

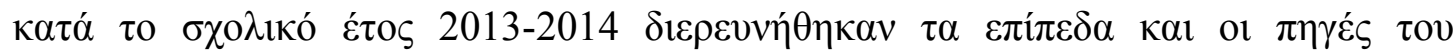

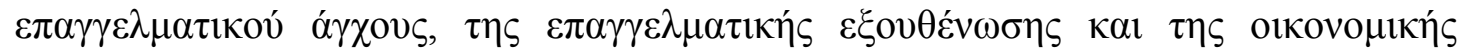

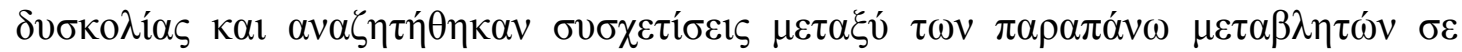

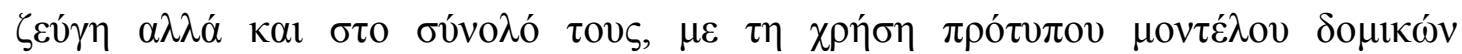

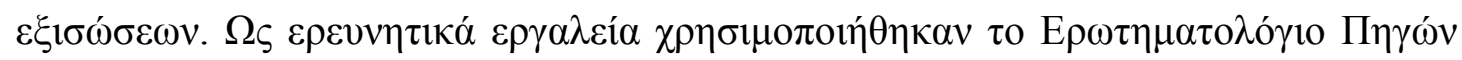

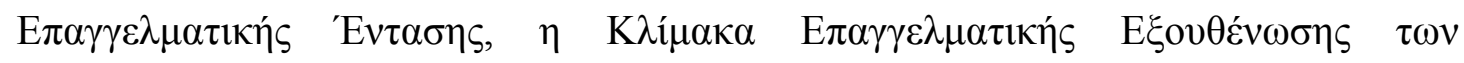

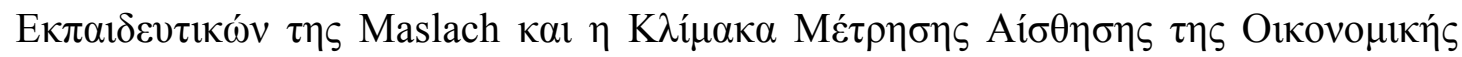

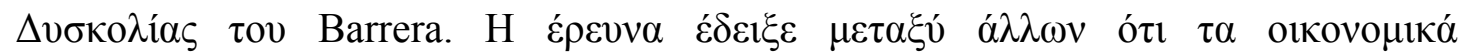

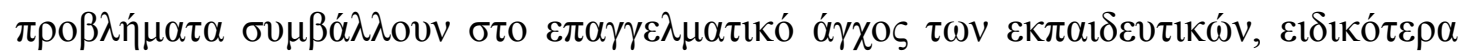

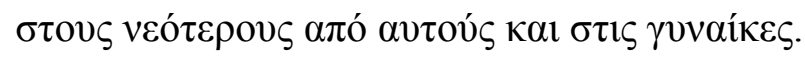

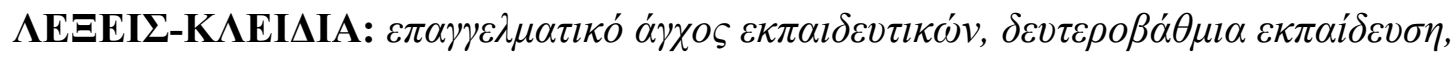

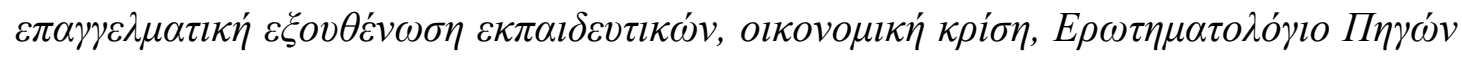

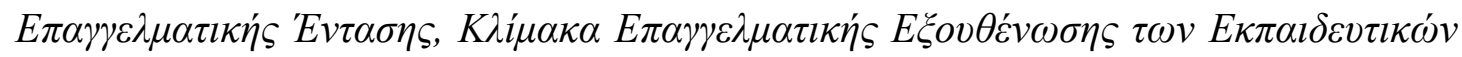

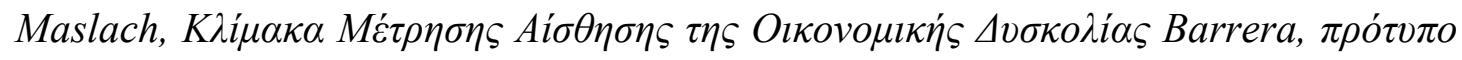

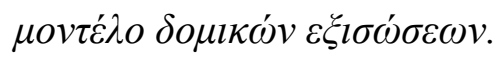




\title{
Occupational stress and professional burnout among Greek teachers in secondary schools under conditions of economic crisis
}

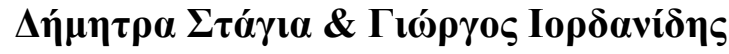

\begin{abstract}
This study examined the levels and sources of teacher stress and burnout, regarding the economic crisis and defined certain correlations between them. Participants were 93 secondary education teachers. The data was collected using the Sources of Occupational Stress Questionnaire, the Maslach Burnout Inventory and Barrera's Perceived Economic Hardship Questionnaire. The research have been held during the school year 2013-2014. The results indicated that the economic crisis has affected the professional stress in many ways, especially for younger teachers and female teachers. The structural equation model revealed that occupational stress, teachers' burnout and economic hardship are interconnected.
\end{abstract}

KEY-WORDS: Occupational stress of teachers, secondary school teachers, teachers' burnout, economic crisis, Sources of Occupational Stress Questionnaire, Maslach Burnout Inventory, Barrera's Perceived Economic Hardship Questionnaire, structural equation model 


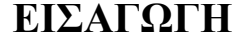

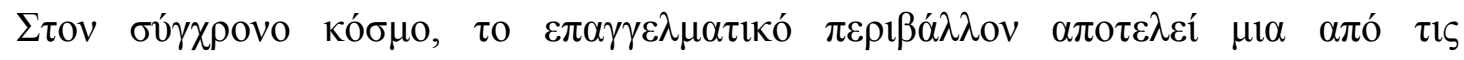

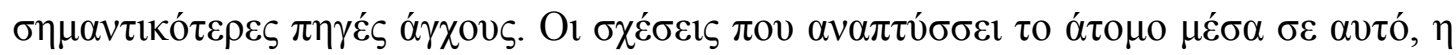

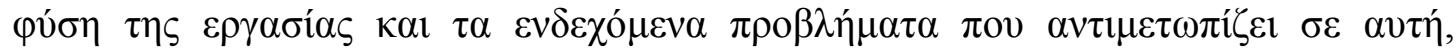

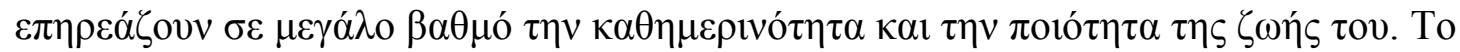

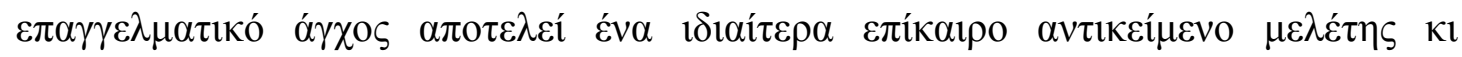

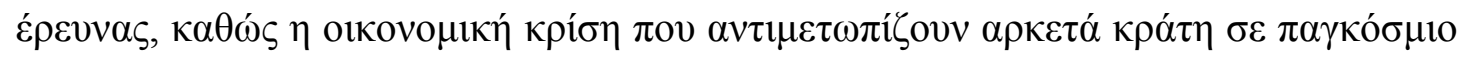

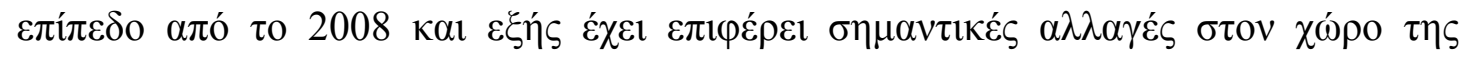

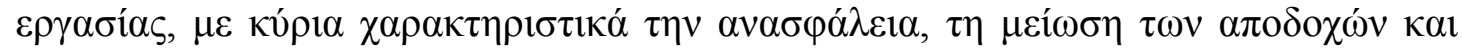

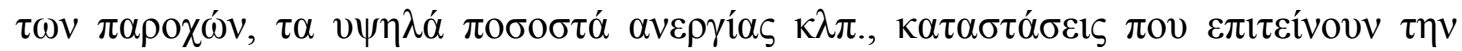

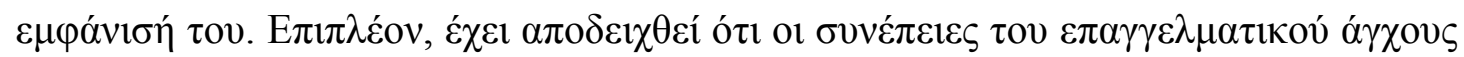

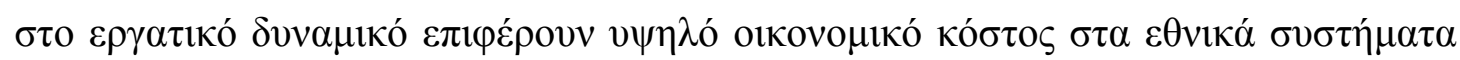

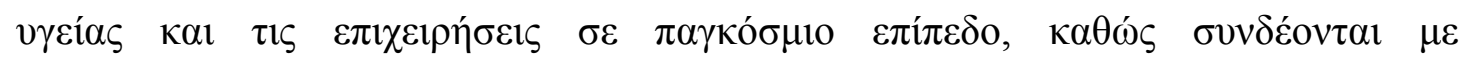

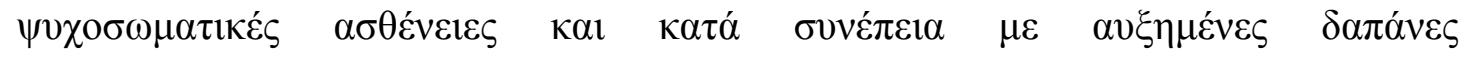

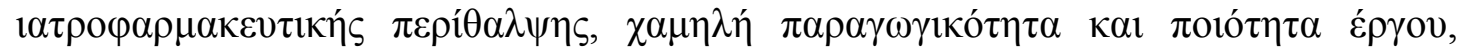

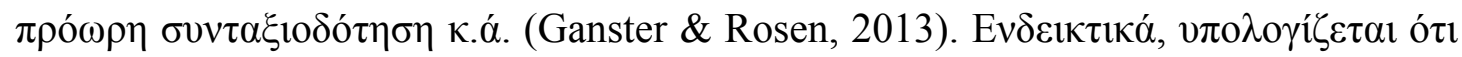

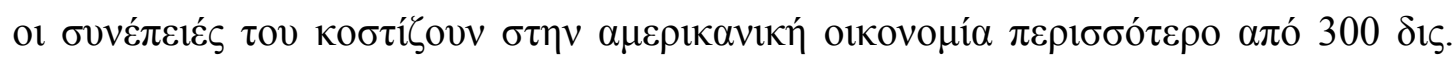

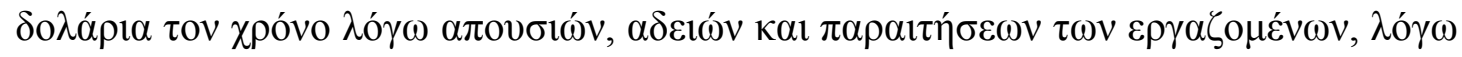

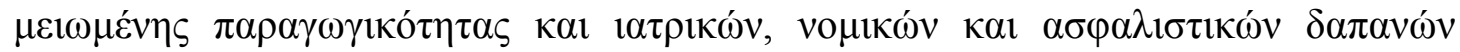

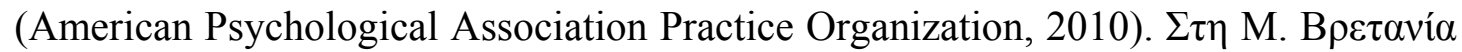

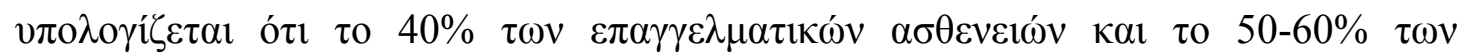

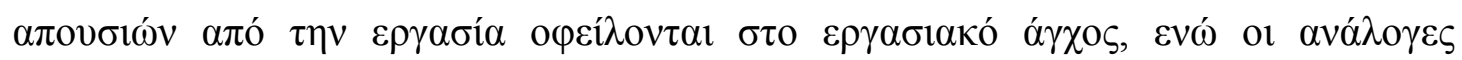

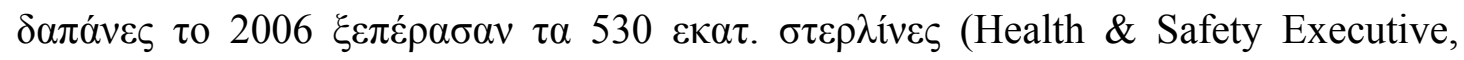




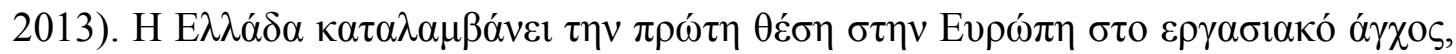
$\mu \varepsilon \pi 0 \sigma 0 \sigma \tau o ́$ 55\% (European Agency for Safety and Health at Work, 2009).

To $\varepsilon \pi \alpha \gamma \gamma \varepsilon \lambda \mu \alpha \tau \imath \kappa o ́ ~ \alpha ́ \gamma \chi o \varsigma \kappa \alpha \iota ~ \eta \varepsilon \xi o v \theta \varepsilon ́ v \omega \sigma \eta \tau o v \varepsilon \kappa \pi \alpha l \delta \varepsilon v \tau \imath \kappa o v ́$

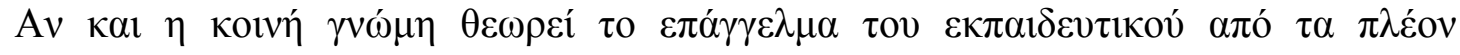

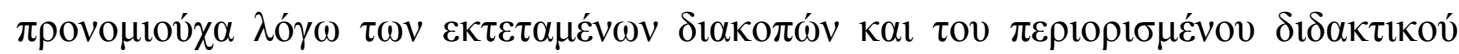

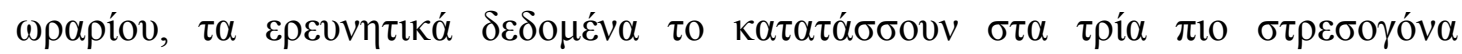
$\varepsilon \pi \alpha \gamma \gamma \varepsilon \dot{\varepsilon} \mu \alpha \tau \alpha \pi \alpha \gamma \kappa o \sigma \mu i ́ \omega \varsigma$ (Johnson et al., 2005 O.E.C.D., 2013a Travers \& Cooper, 1993).

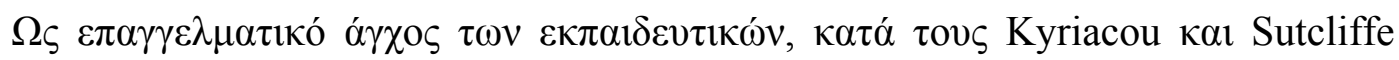

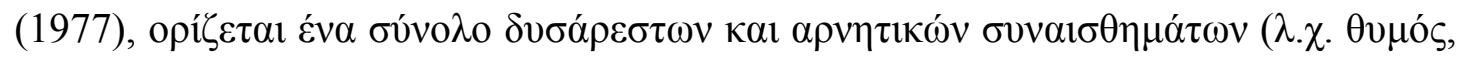

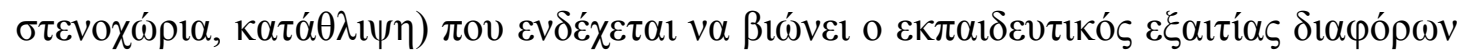

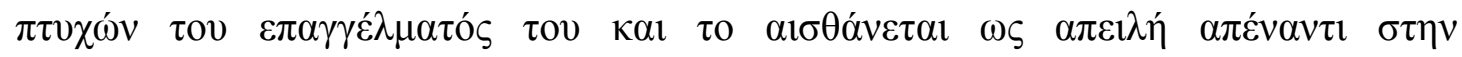

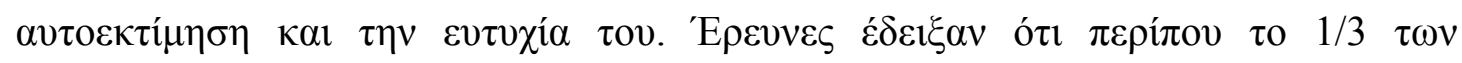

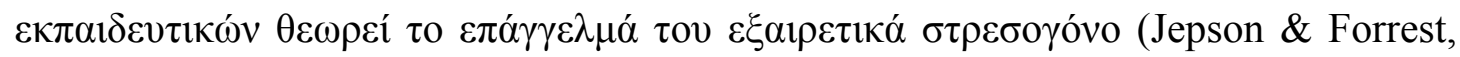

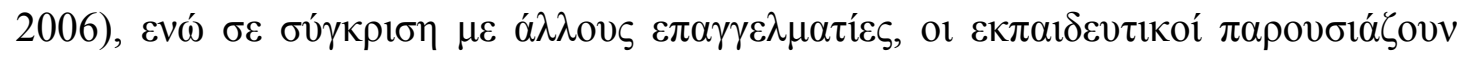

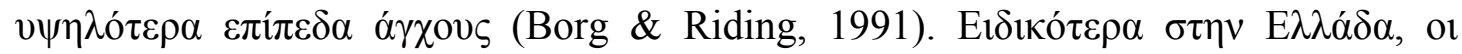

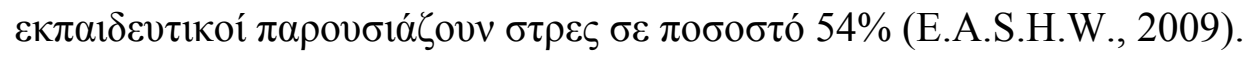

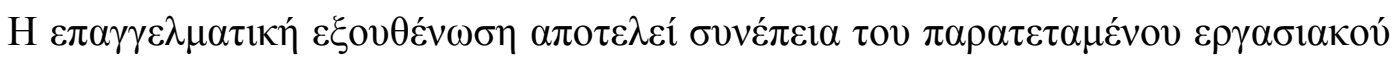

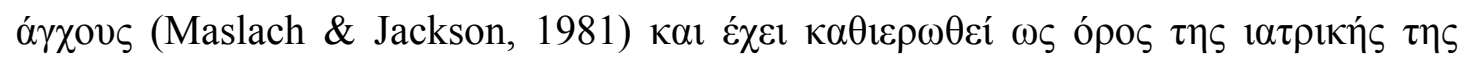

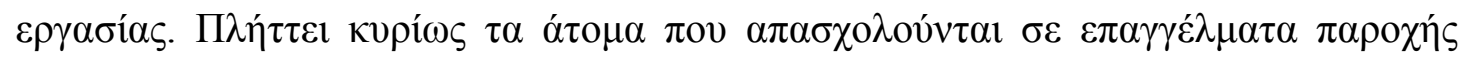

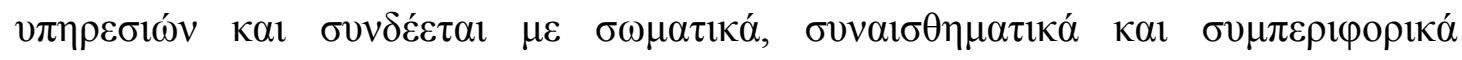

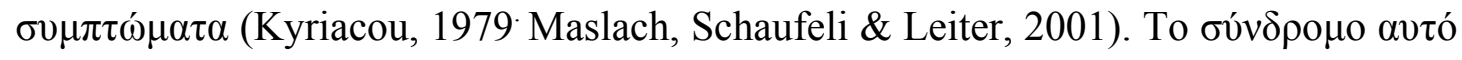

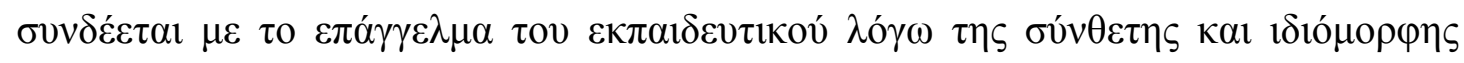

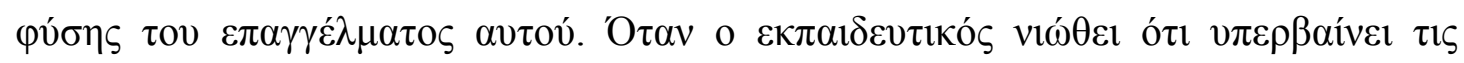

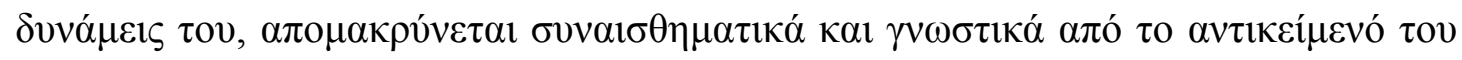




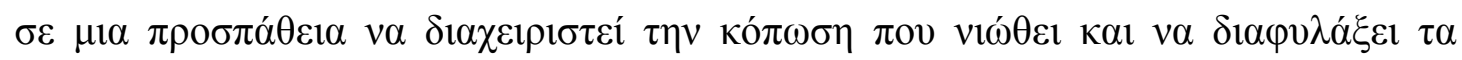

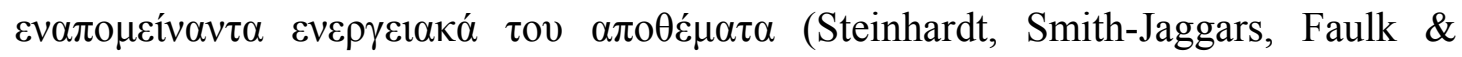
Gloria, 2011).

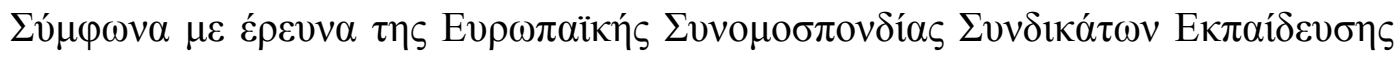

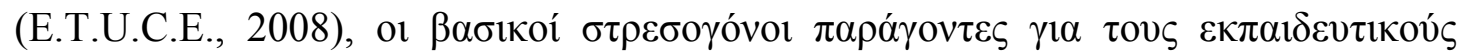

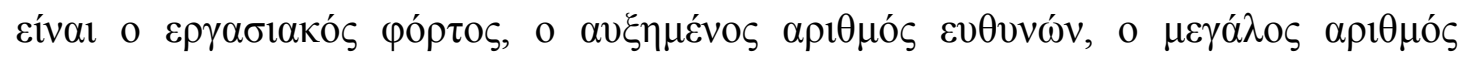

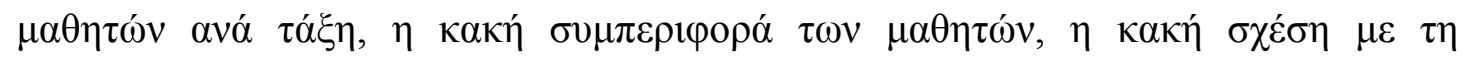

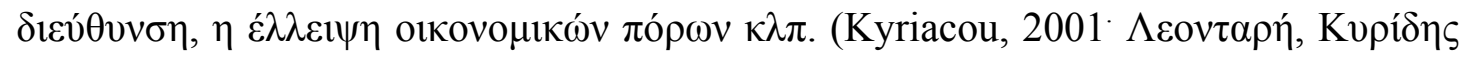

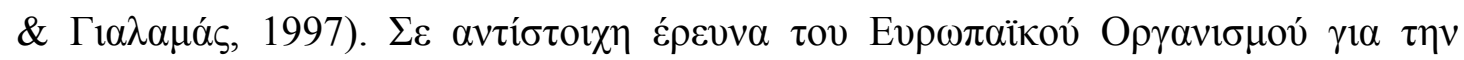

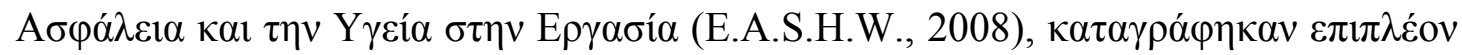

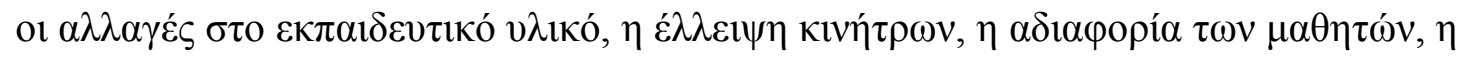

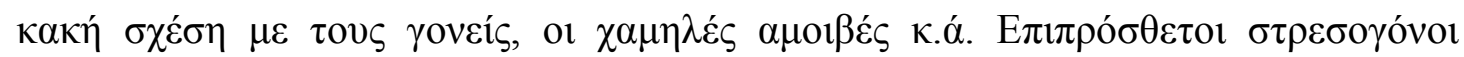

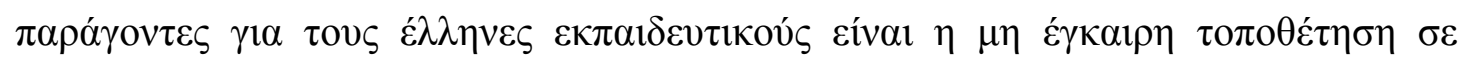

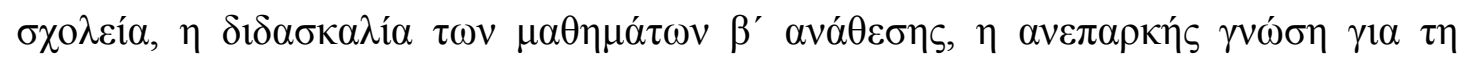

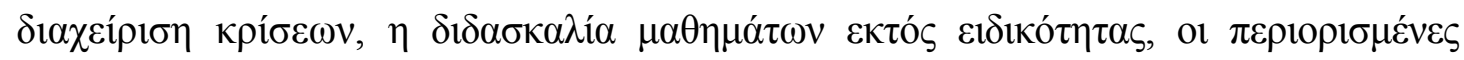

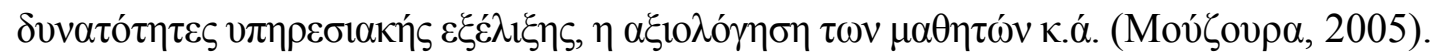

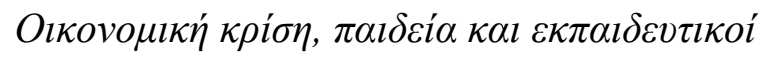

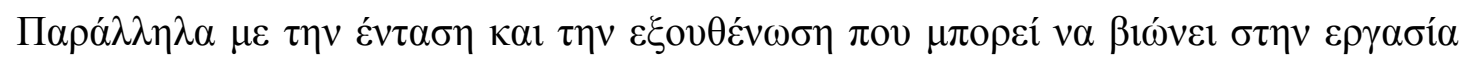

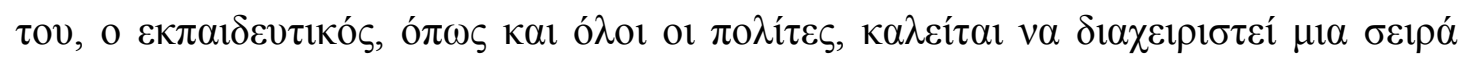

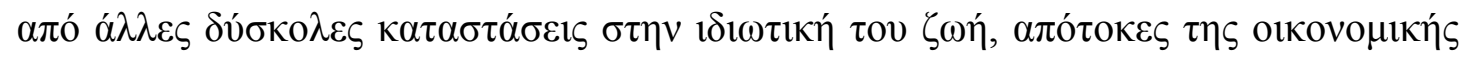

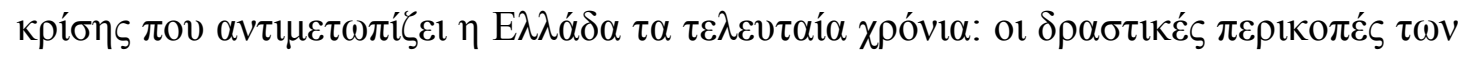

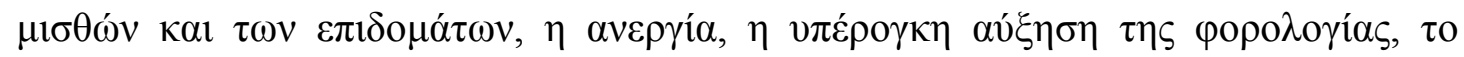

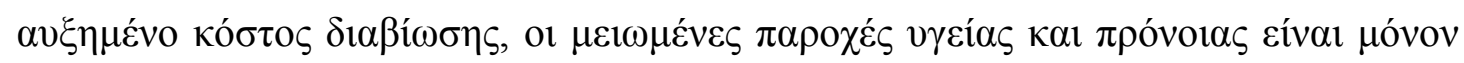

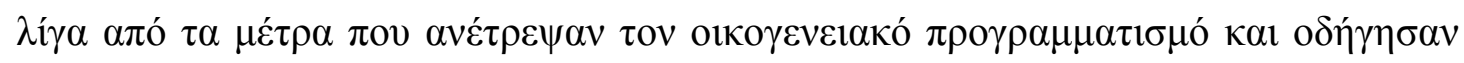

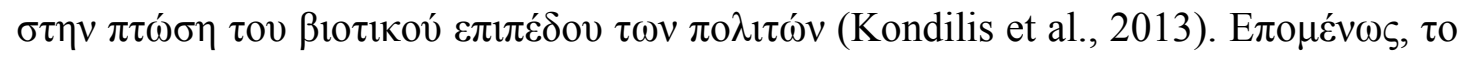




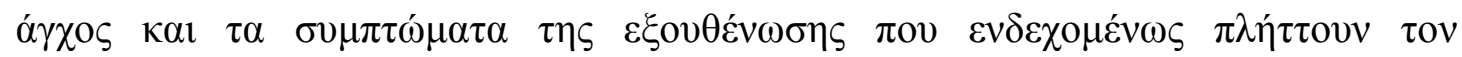

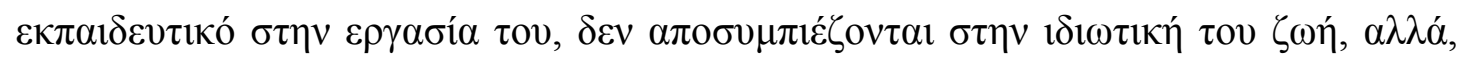
$\alpha \nu \tau i ́ \theta \varepsilon \tau \alpha, \delta 10 \gamma \kappa \omega ́ v o v \tau \alpha$.

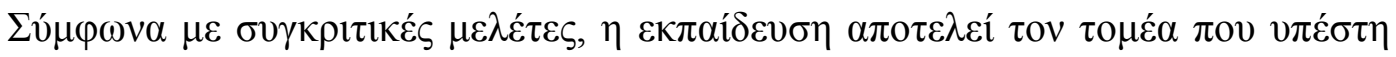

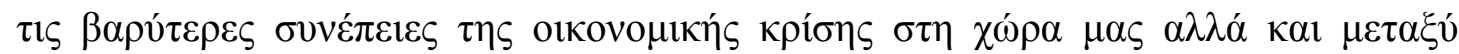

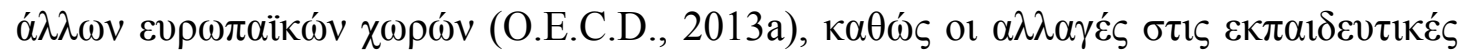

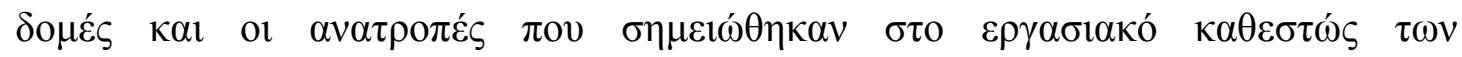

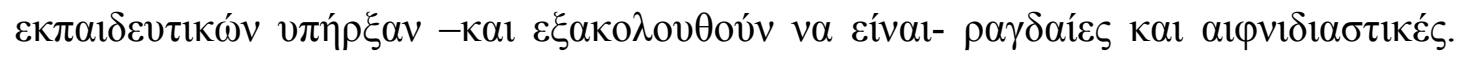

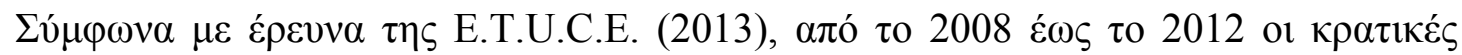

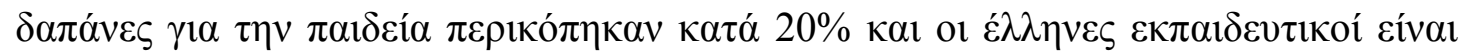

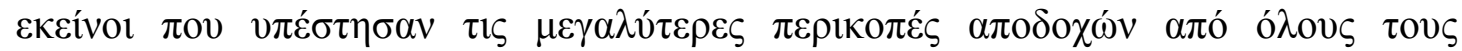

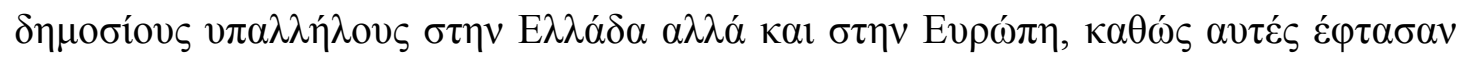

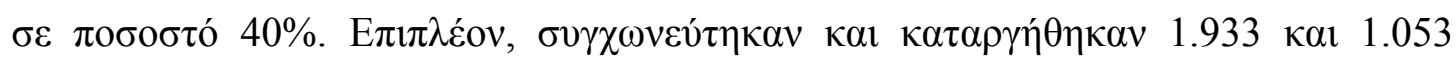

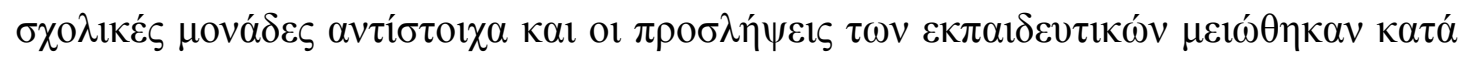

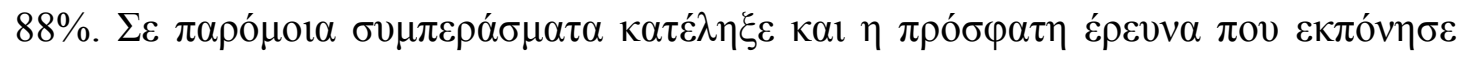

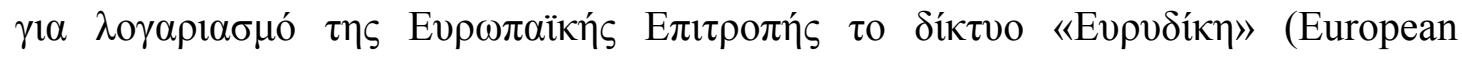

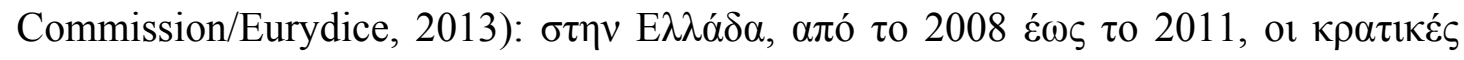

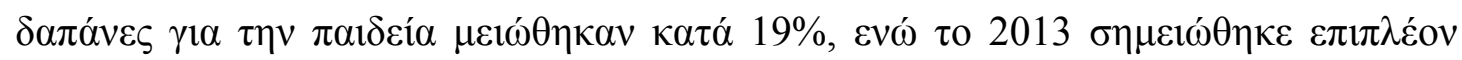

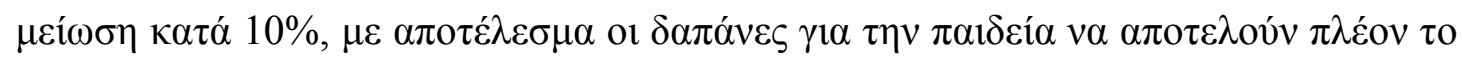

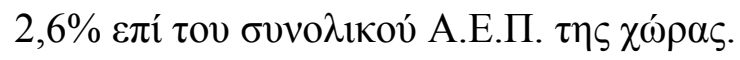

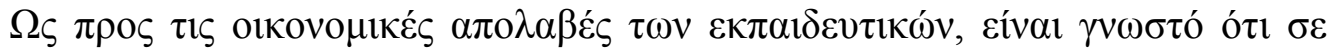

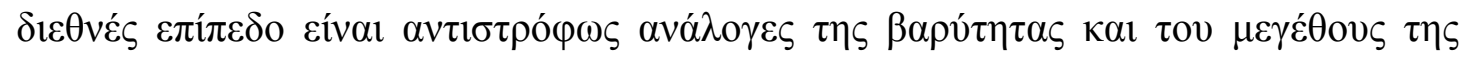

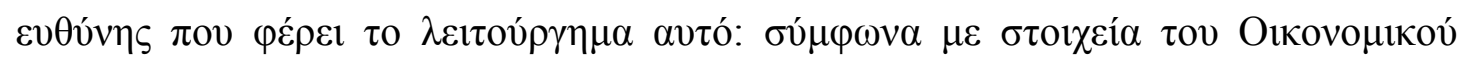

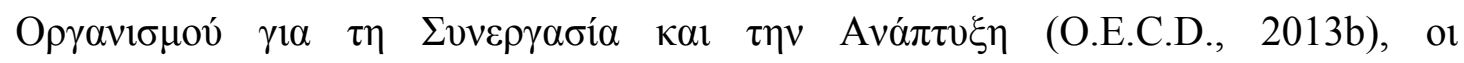

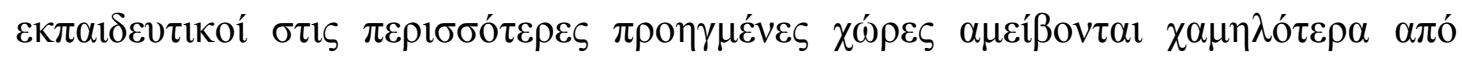




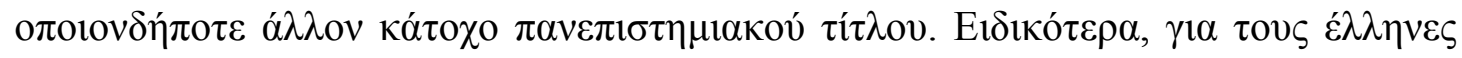

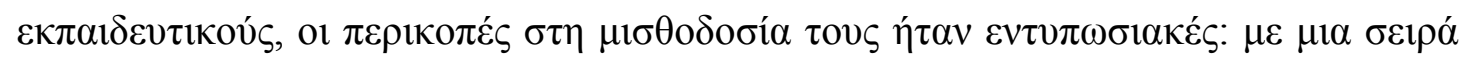

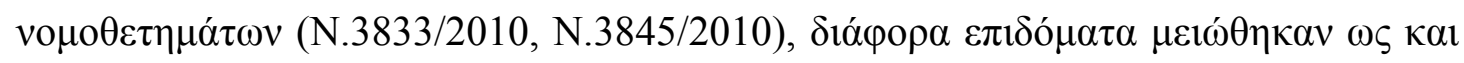

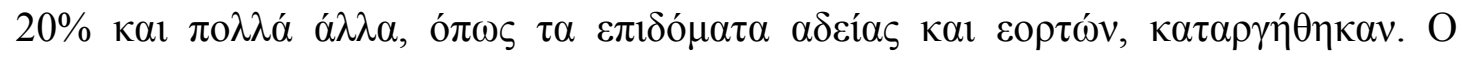

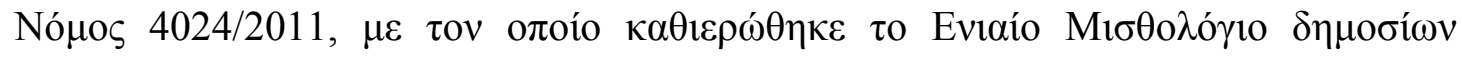

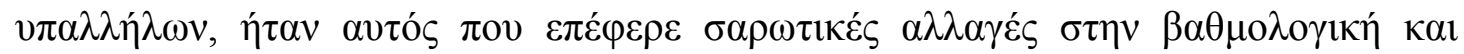

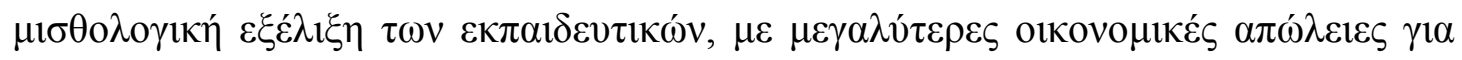

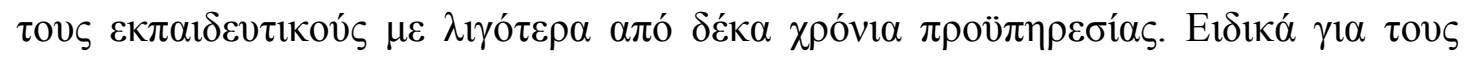

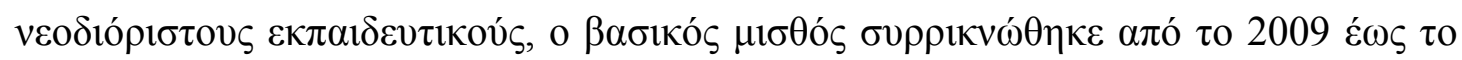

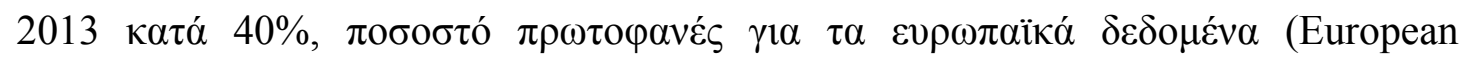
Commission/Eurydice, 2013).

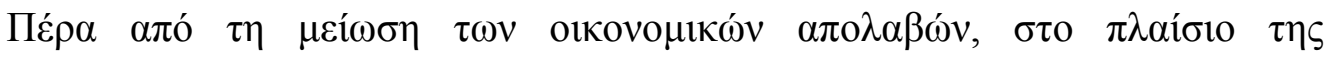

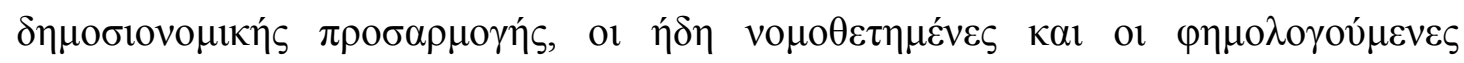

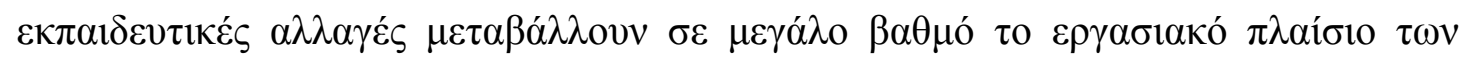

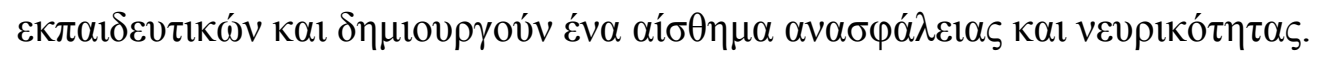




\section{$\operatorname{ME\Theta O} \Delta 0 \Sigma$}

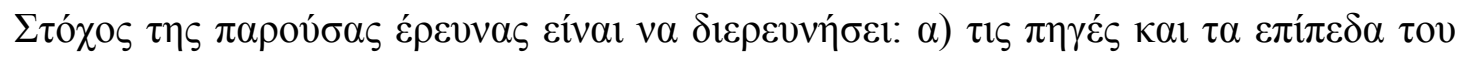

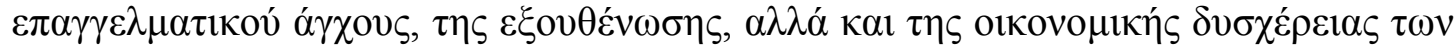

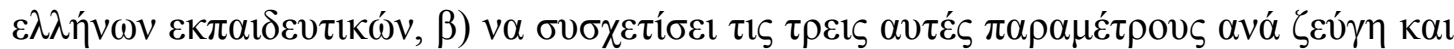

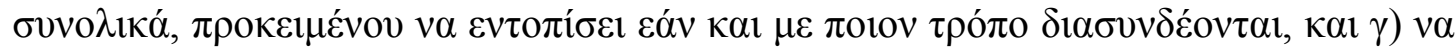

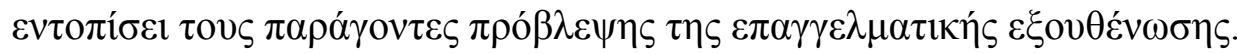

\section{$\Delta \varepsilon i ́ \gamma \mu \alpha$}

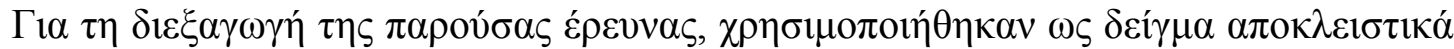

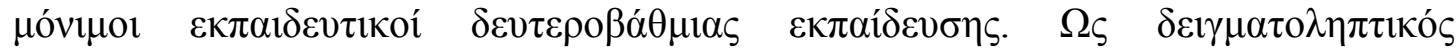

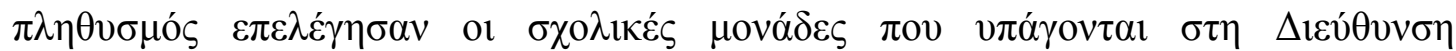

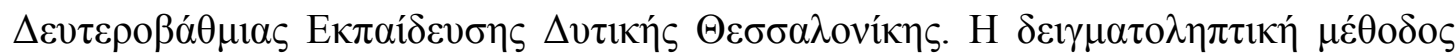

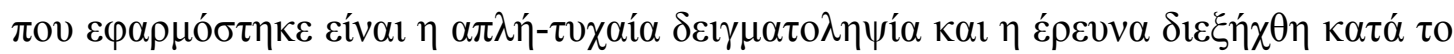

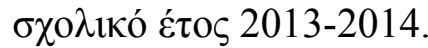

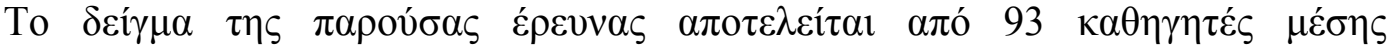

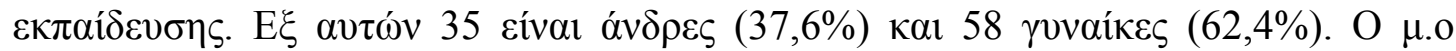

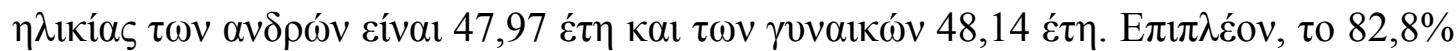

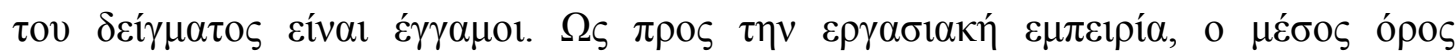

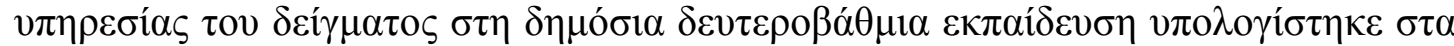
$18,85 \varepsilon \dot{\varepsilon} \tau$.

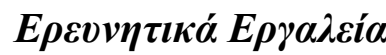

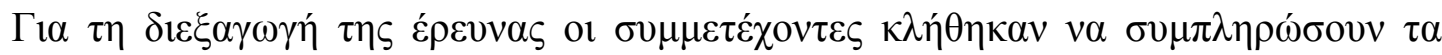
$\varepsilon \xi \dot{\eta} \varsigma \varepsilon \rho \omega \tau \eta \mu \alpha \tau 0 \lambda o ́ \gamma 1 \alpha:$ 


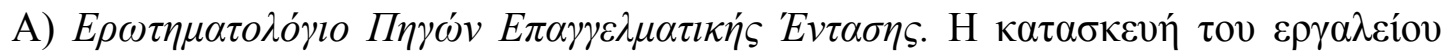

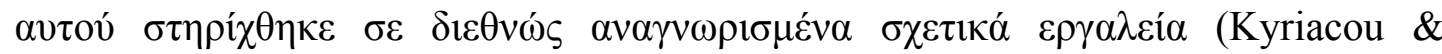

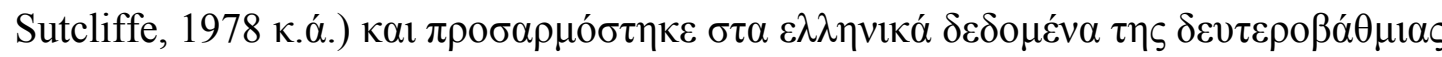

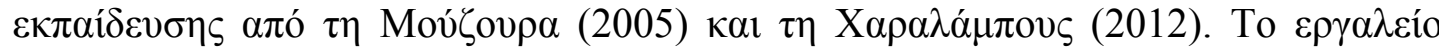

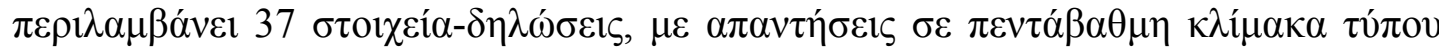

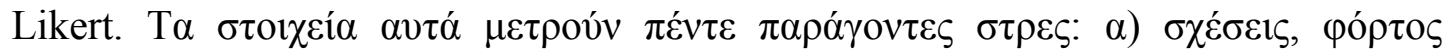

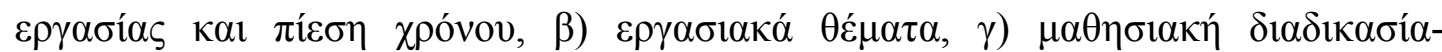

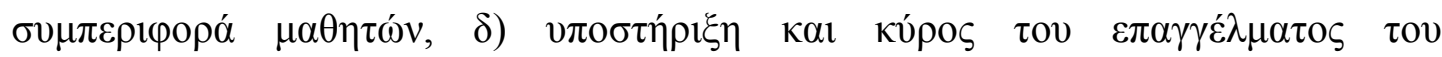

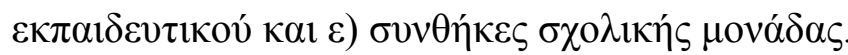

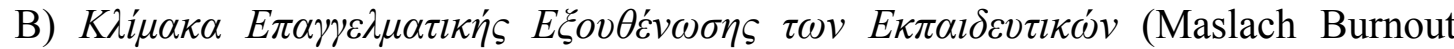

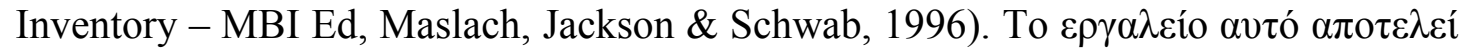

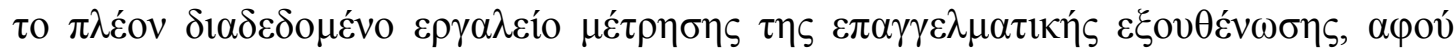

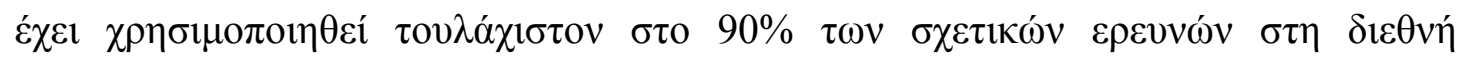

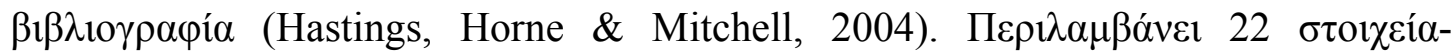

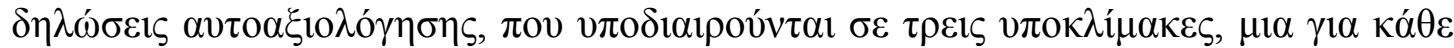

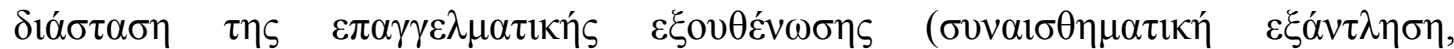

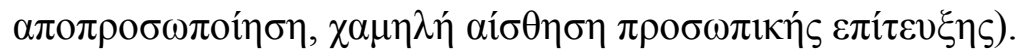

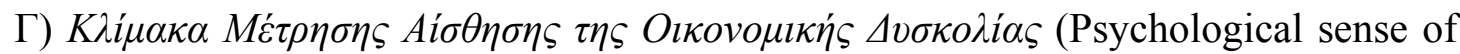

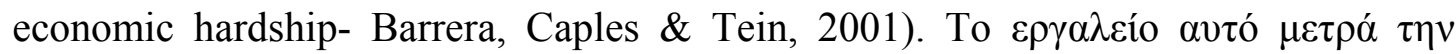

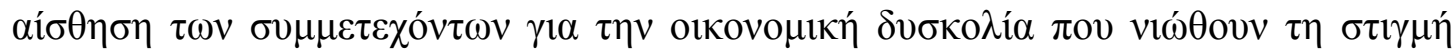

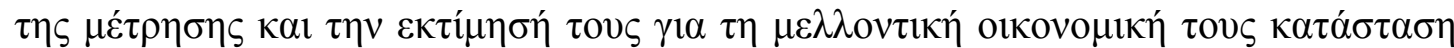

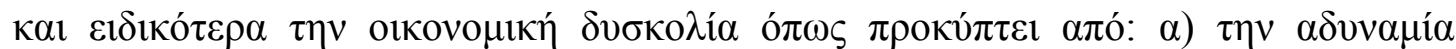

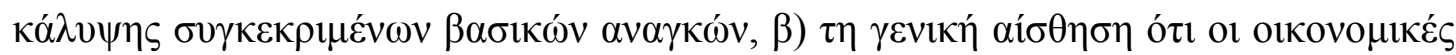

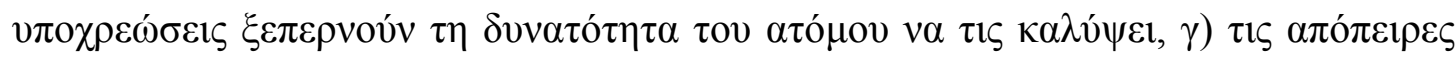




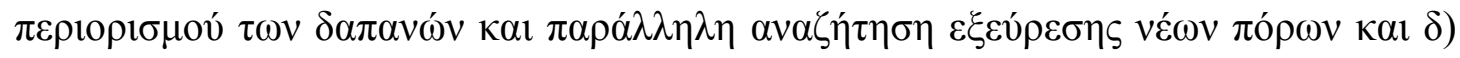

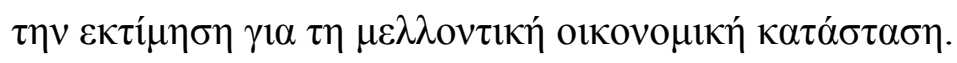

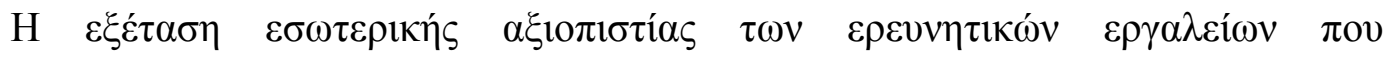

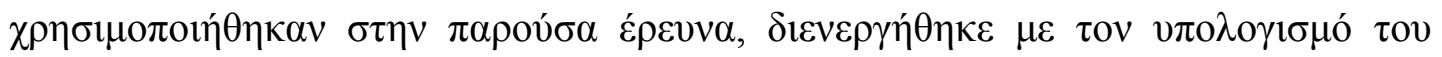

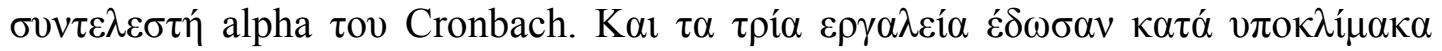

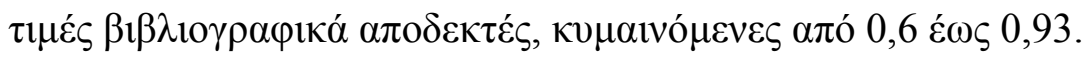




\section{AПOTЕ $\Lambda$ ЕМАТА}

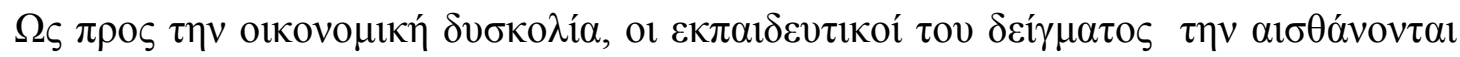

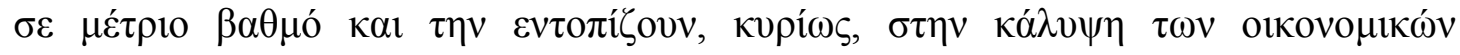

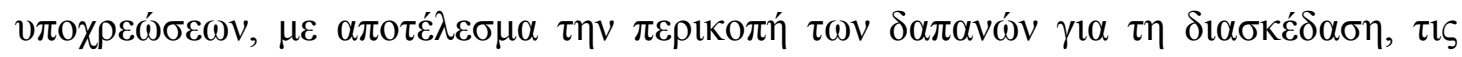

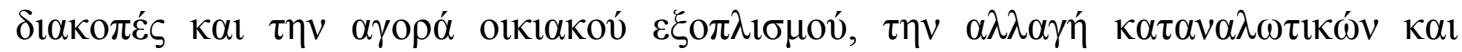

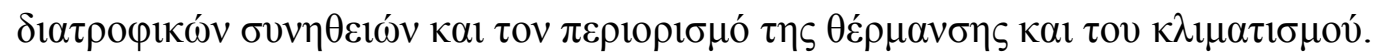

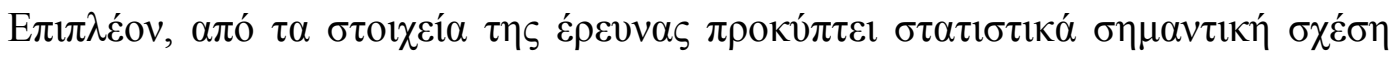

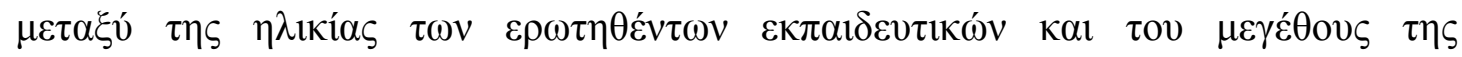

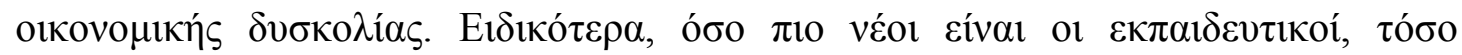

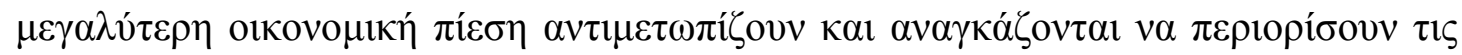

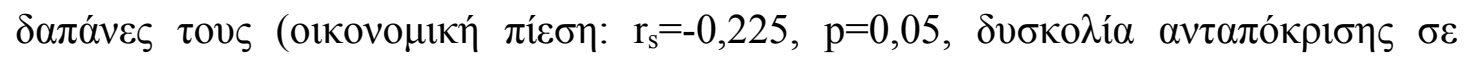

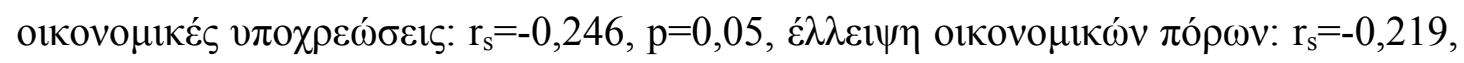
$\left.\mathrm{p}=0,05, \pi \varepsilon \rho 1 \kappa о \pi \varepsilon ́ \varsigma \delta \alpha \pi \alpha \nu \omega ́ v: \mathrm{r}_{\mathrm{s}}=-0,320, \mathrm{p}=0,05\right)$.

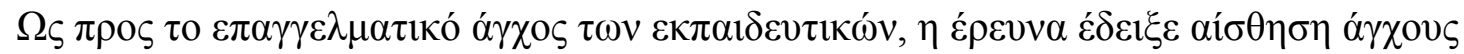

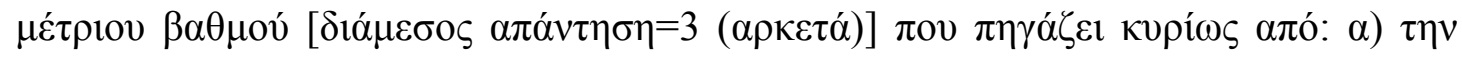

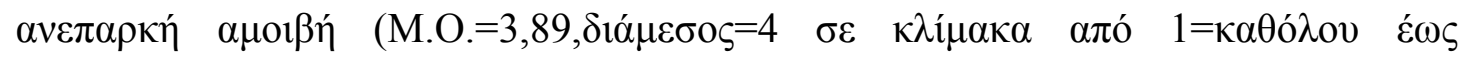

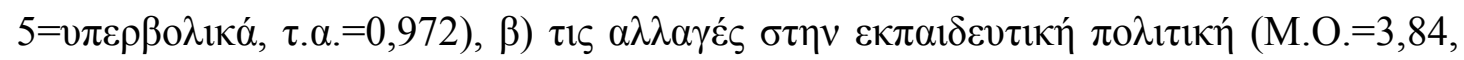

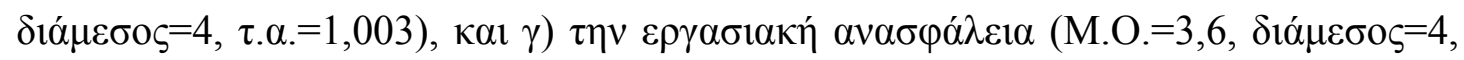
$\tau . \alpha=1,336)$.

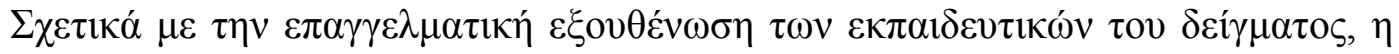

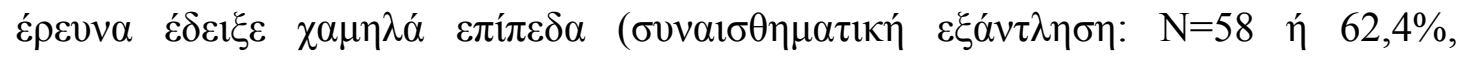

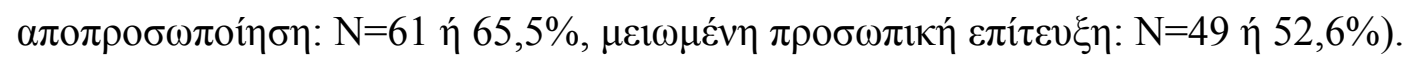

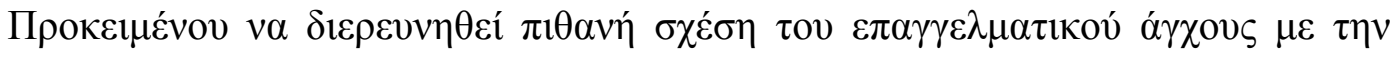

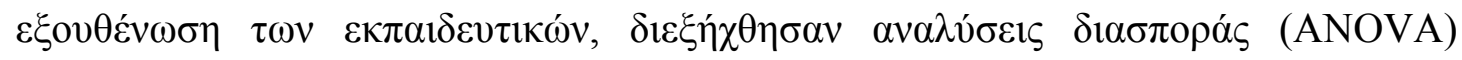




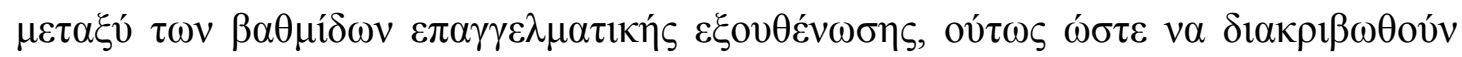

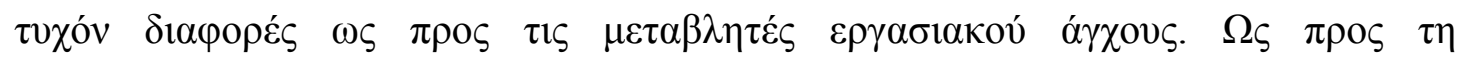

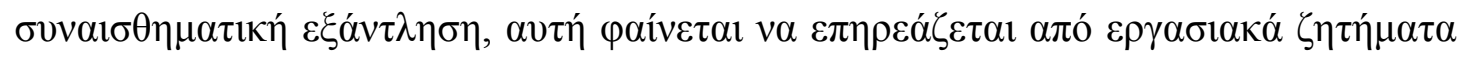

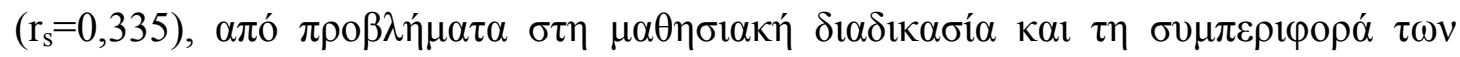

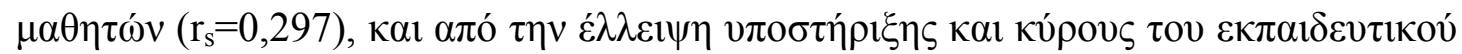

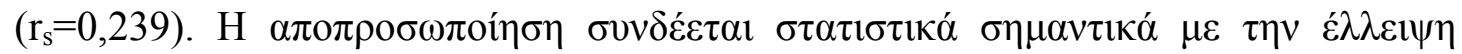

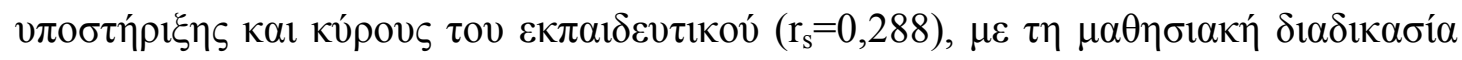

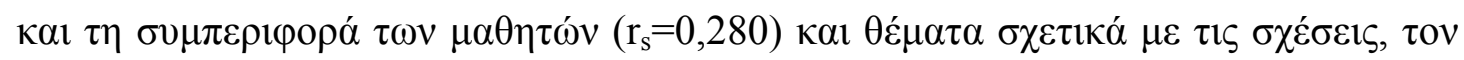

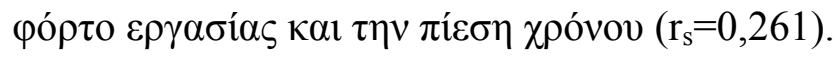

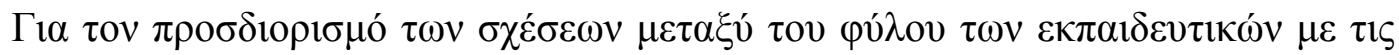

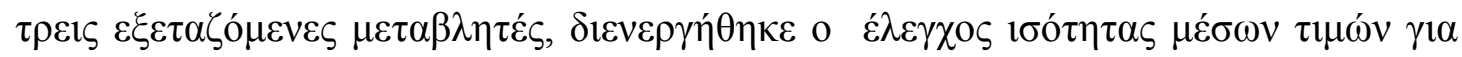

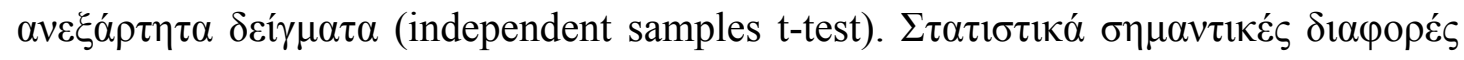

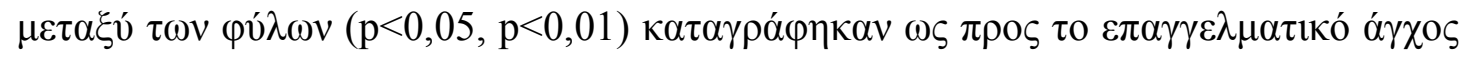

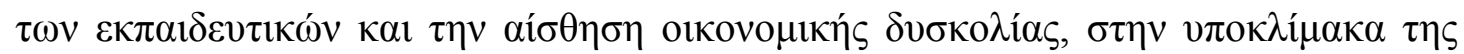

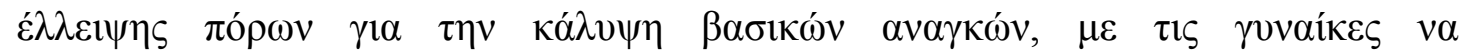

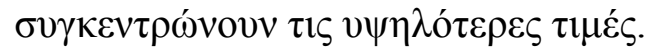

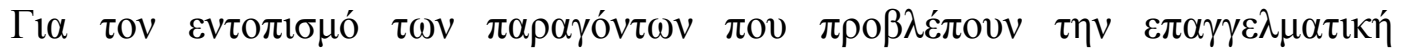

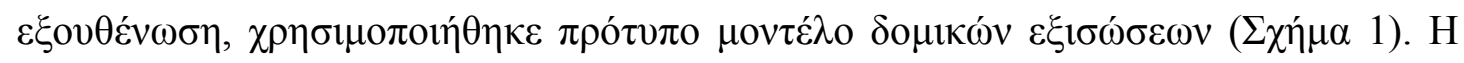

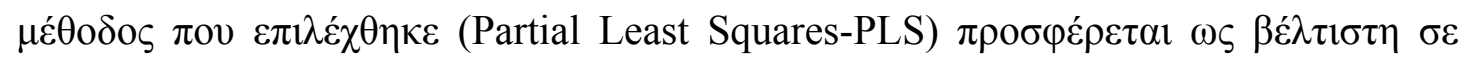

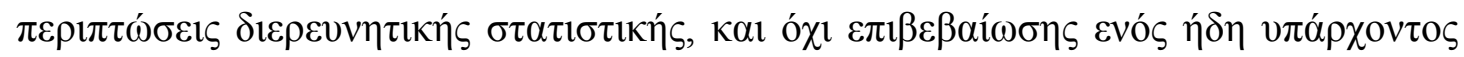

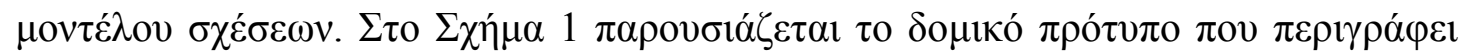

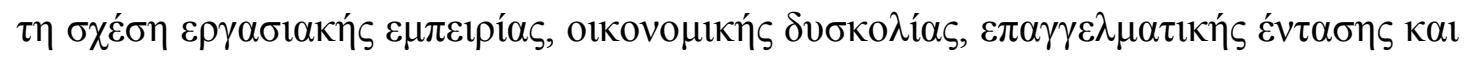

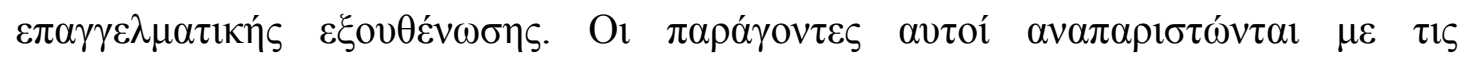

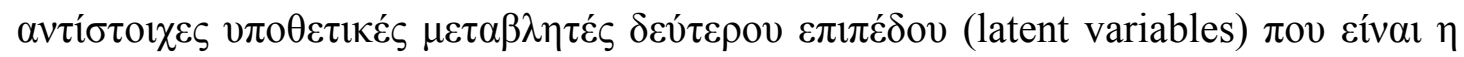

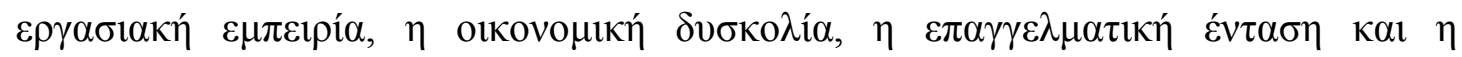




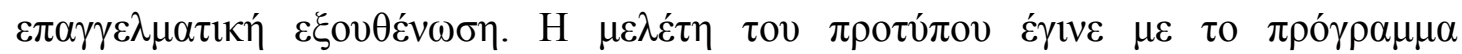
SmartPLS 2.0.

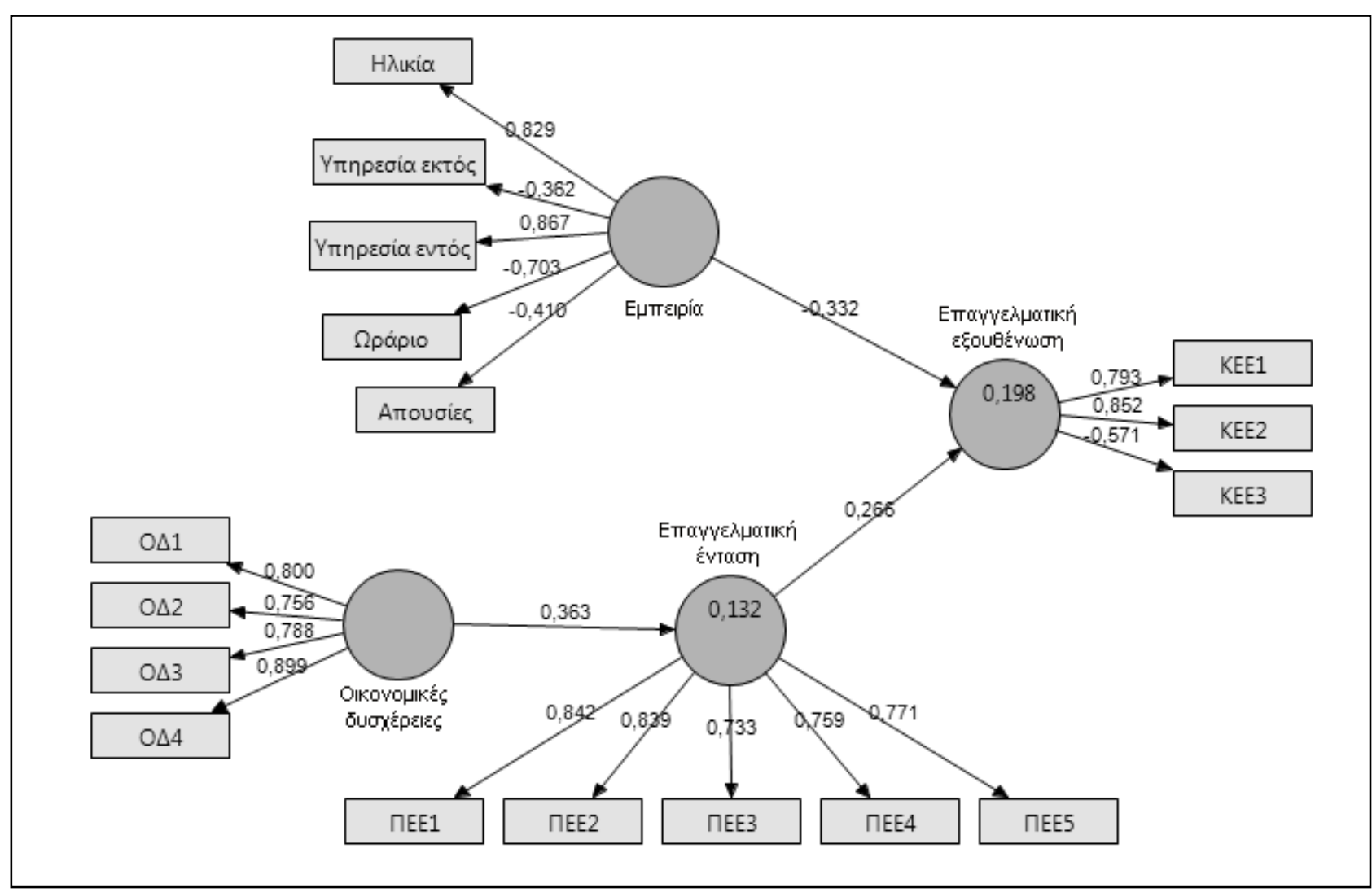

$\Sigma \chi \eta ́ \mu \alpha 1$

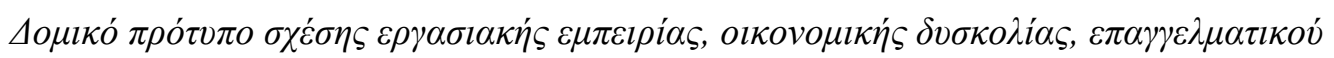

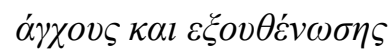

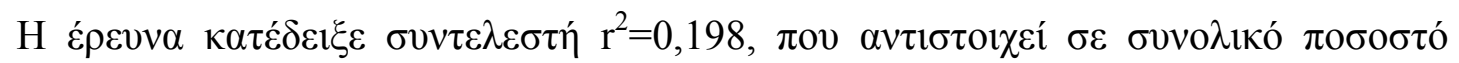

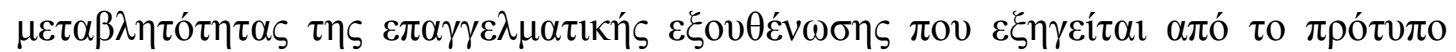

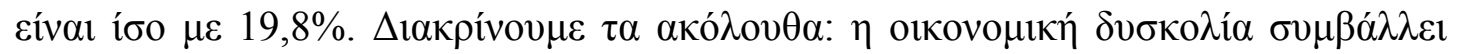

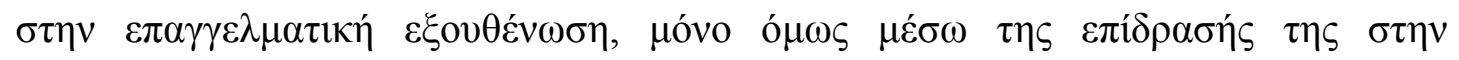

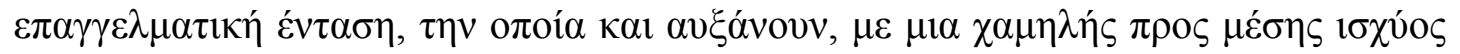

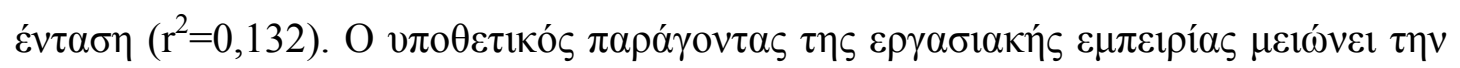




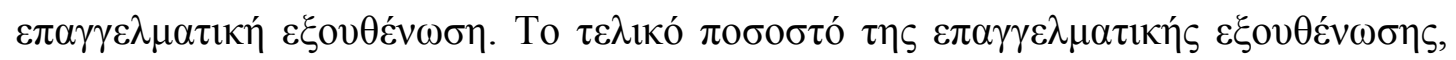

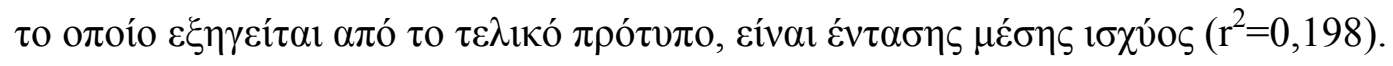




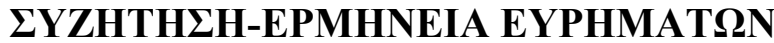

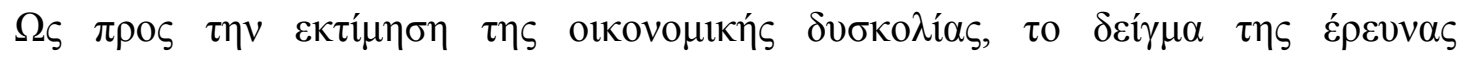

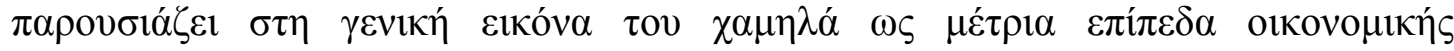

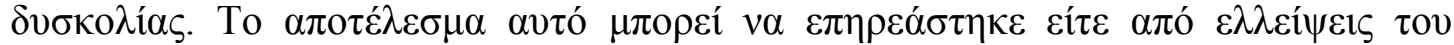

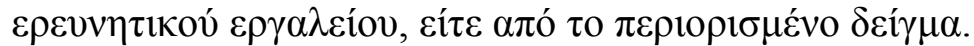

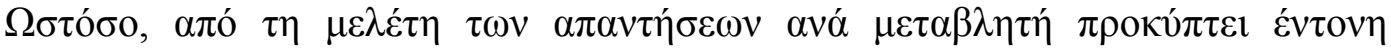

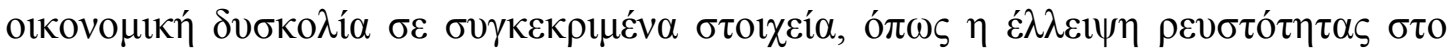

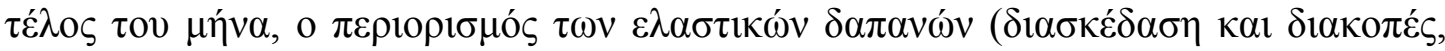

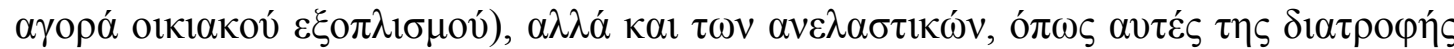

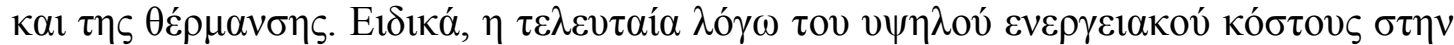

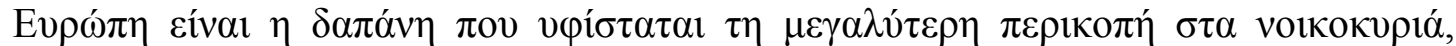

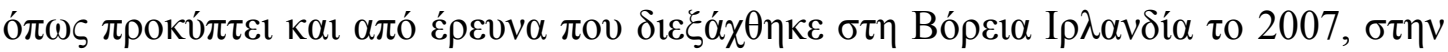

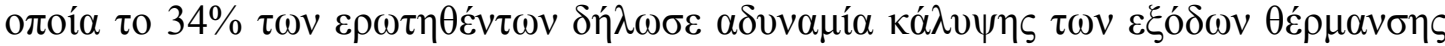
(Hossain et al., 2011).

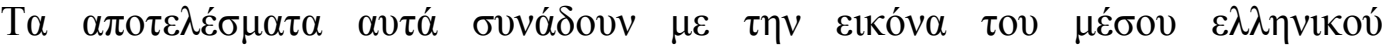

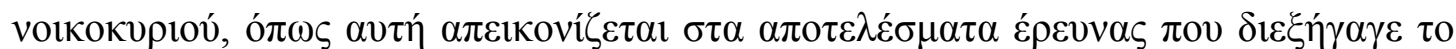

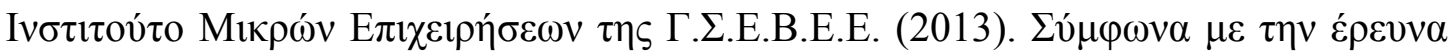

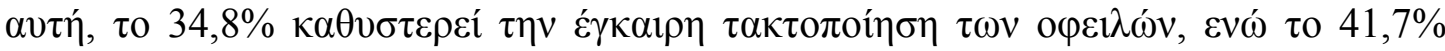

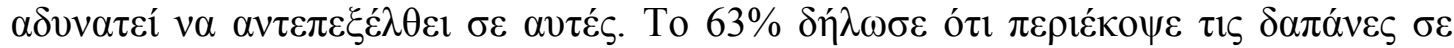

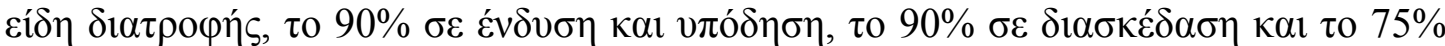

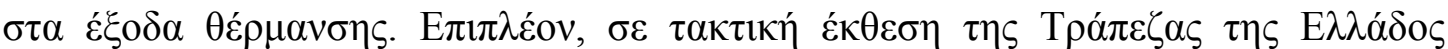

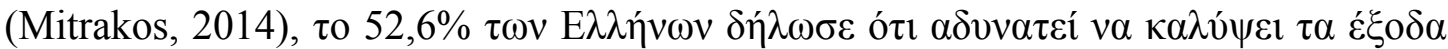
$\theta \varepsilon \dot{p} \mu \alpha v \sigma \eta \varsigma$.

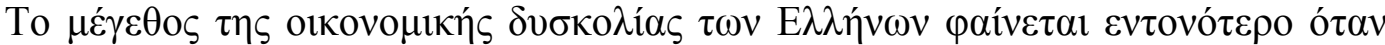




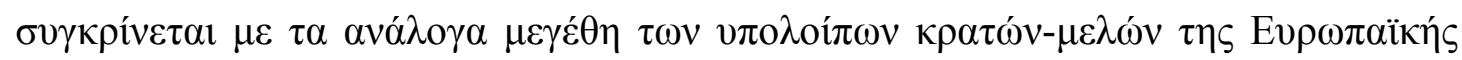

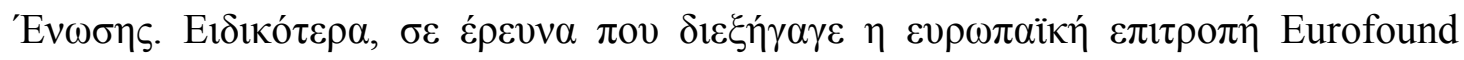

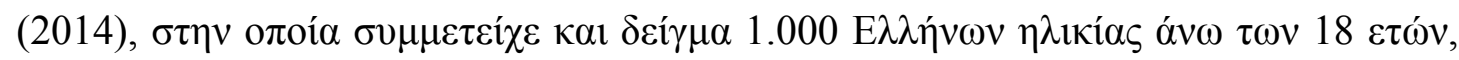

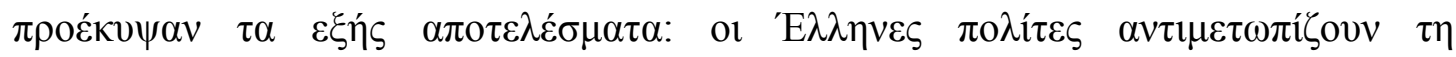

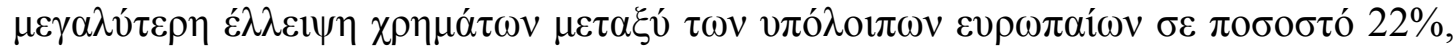

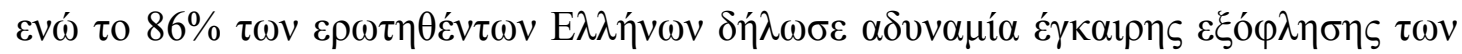

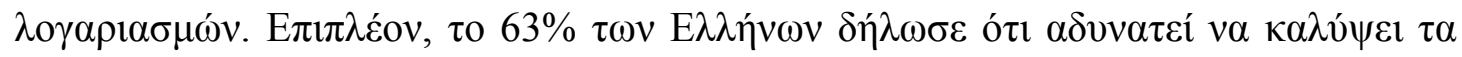

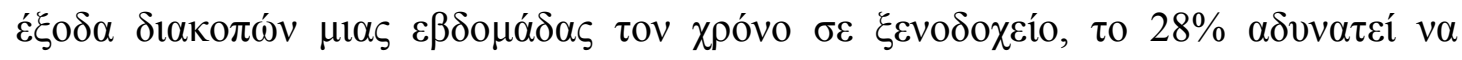

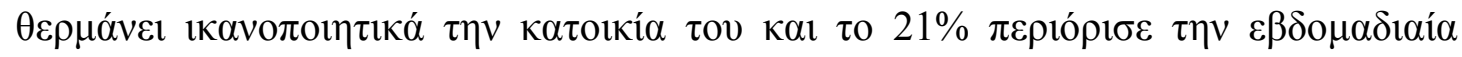

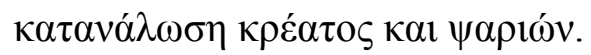

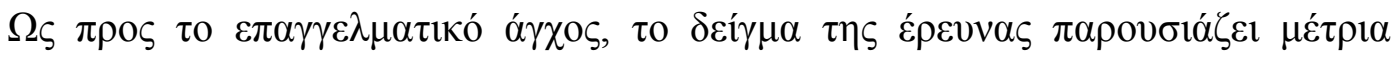

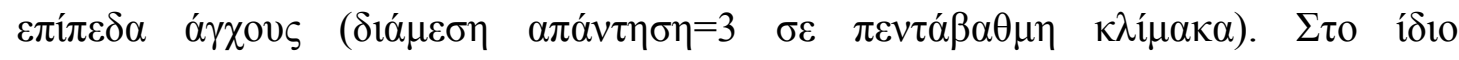

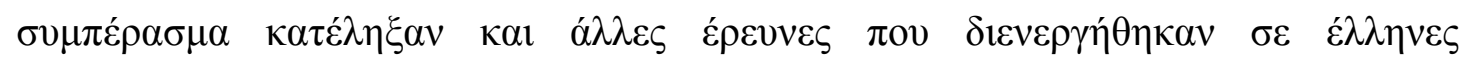

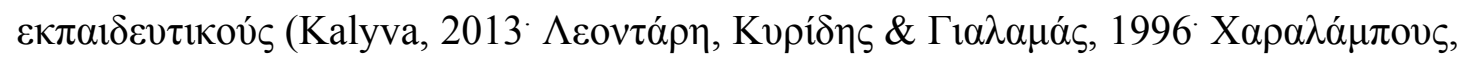

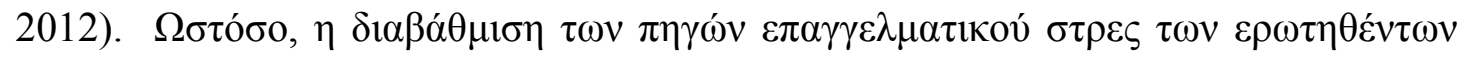

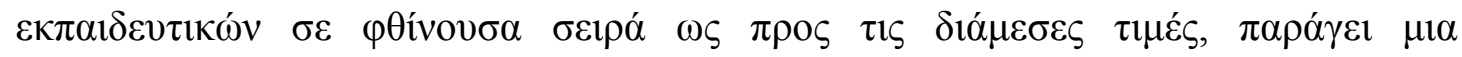

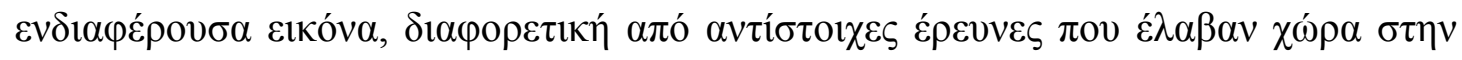

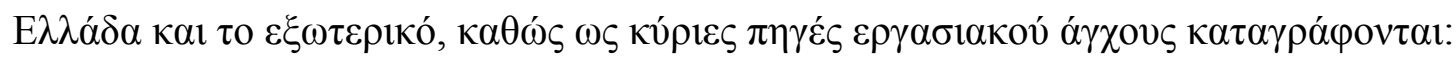

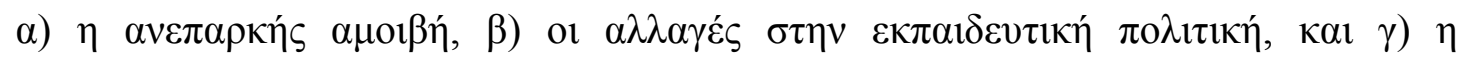

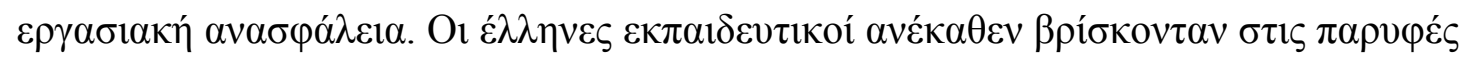

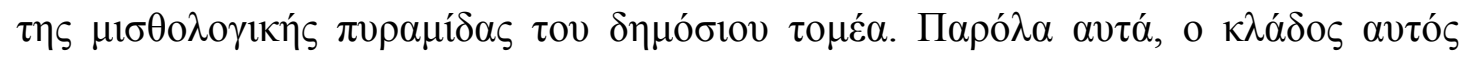

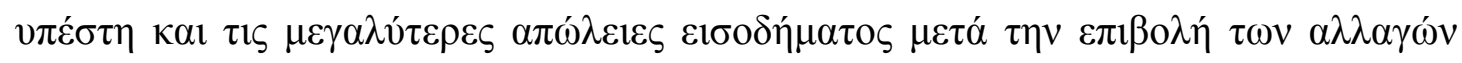

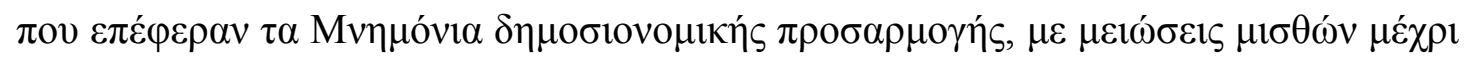

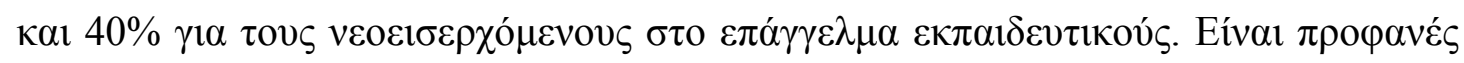

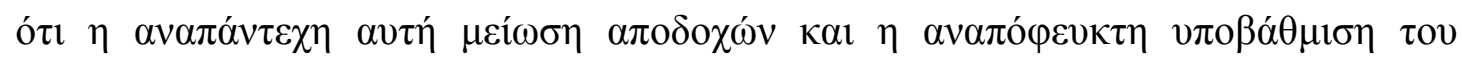




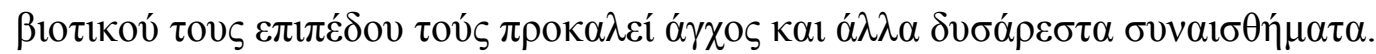

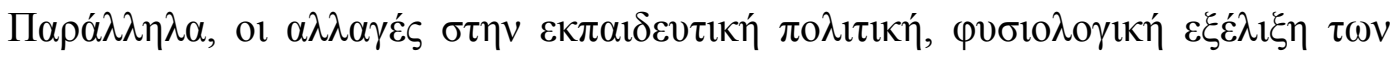

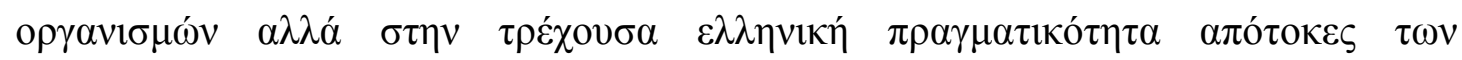

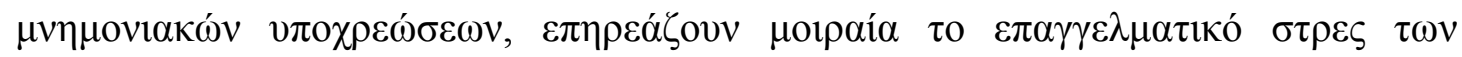

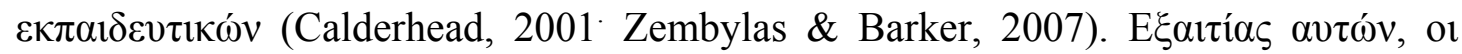

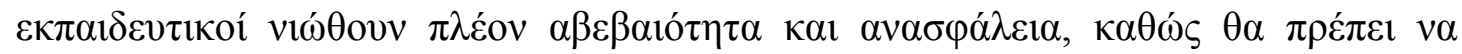

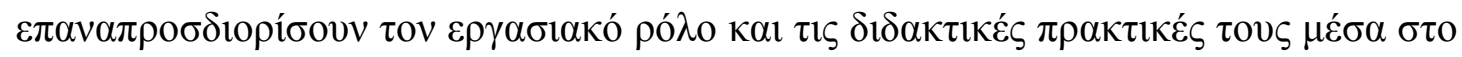

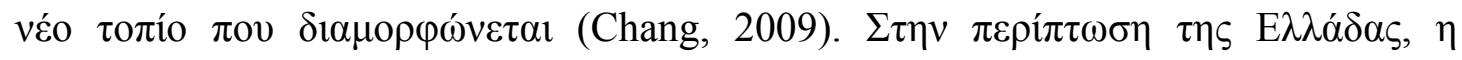

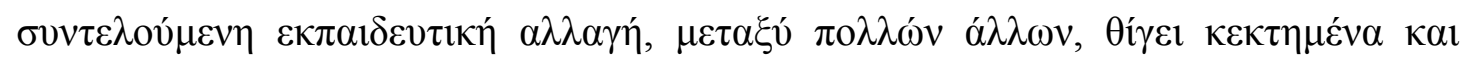

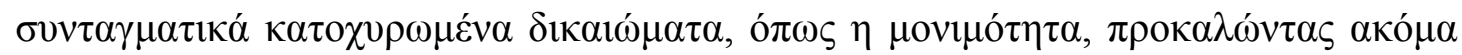

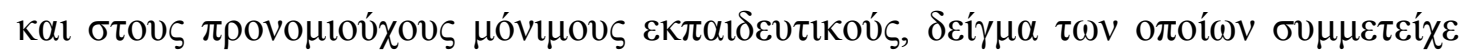

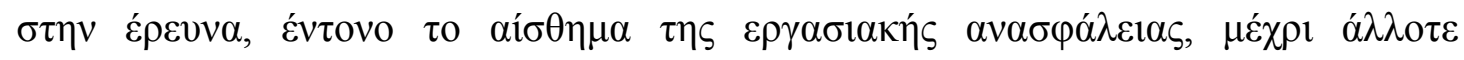

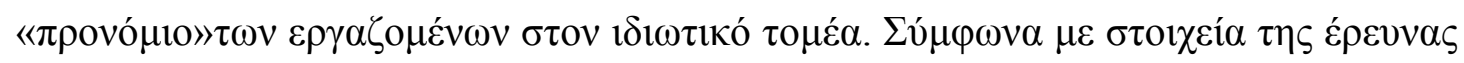

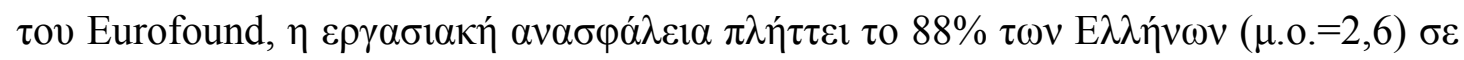

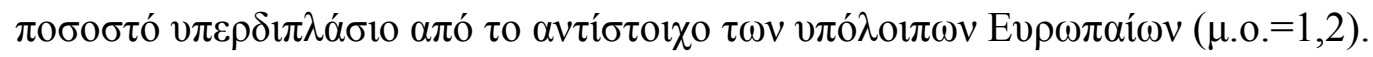

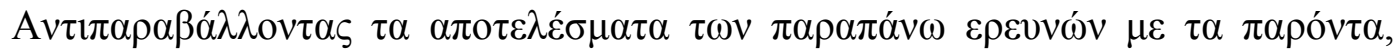

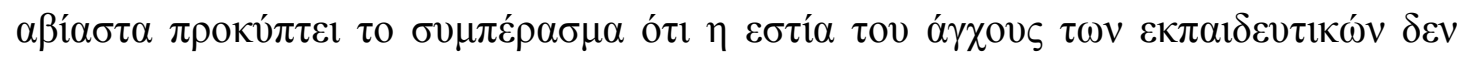

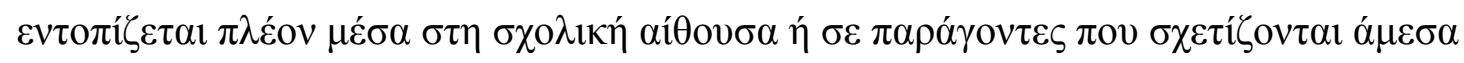

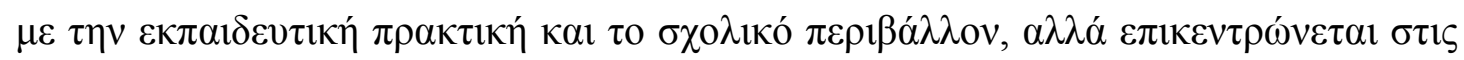

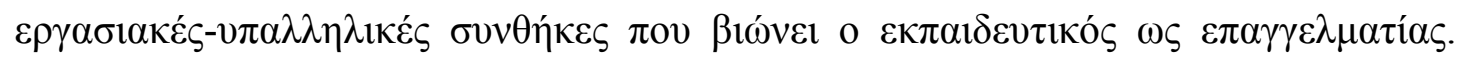

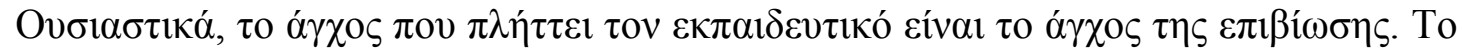

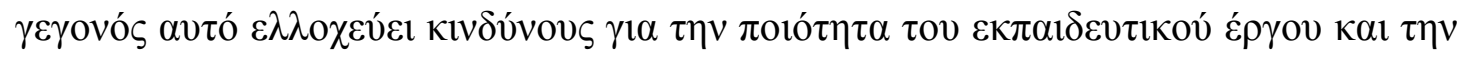

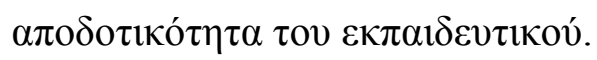

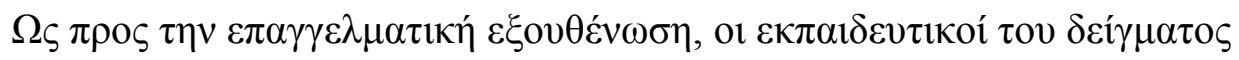

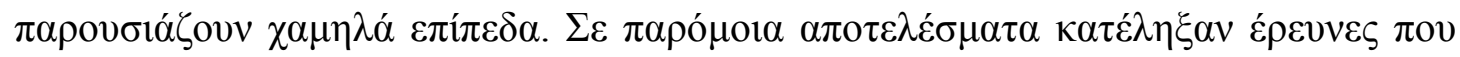




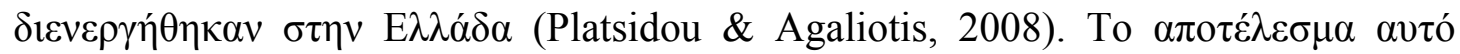

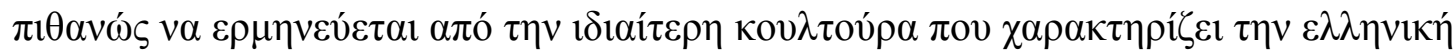

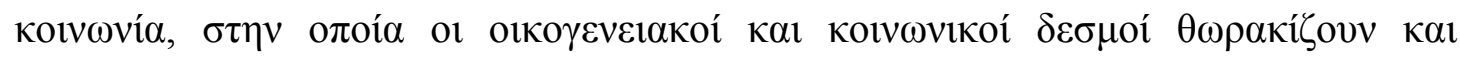

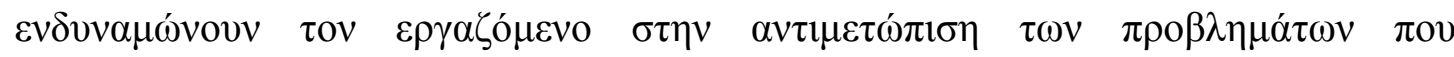

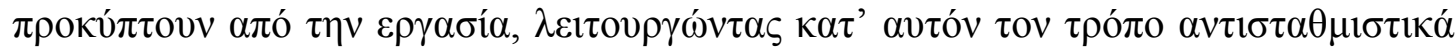

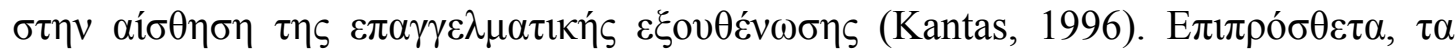

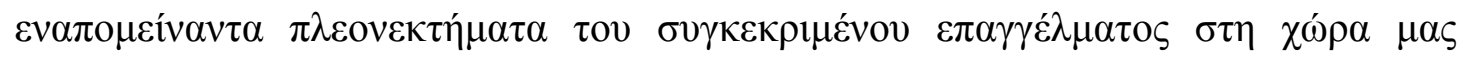

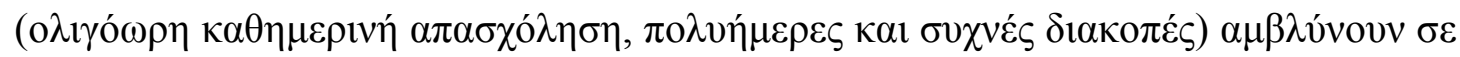

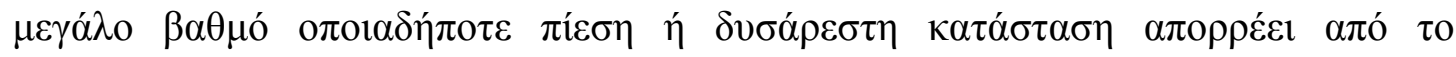
$\varepsilon \rho \gamma \alpha \sigma i \alpha \kappa o ́ ~ \pi \varepsilon \rho ı \beta \alpha ́ \lambda \lambda o v$.

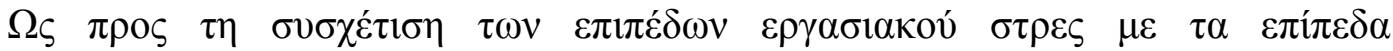

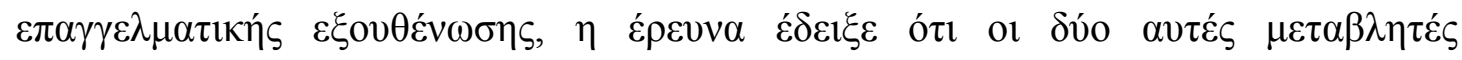

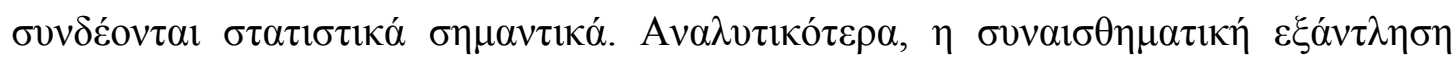

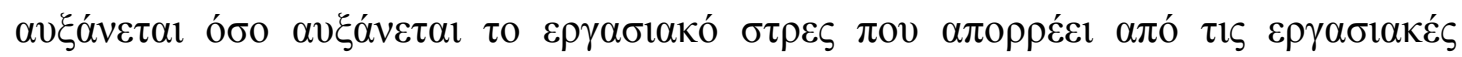

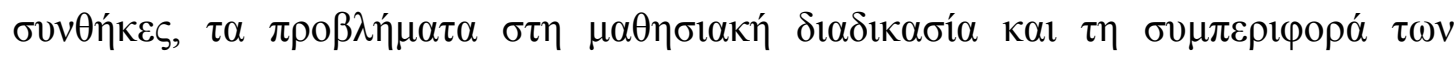

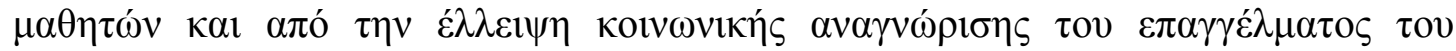

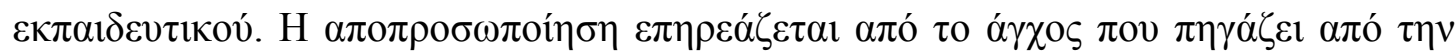

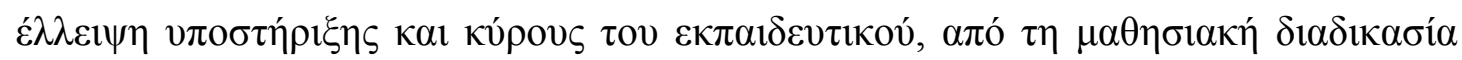

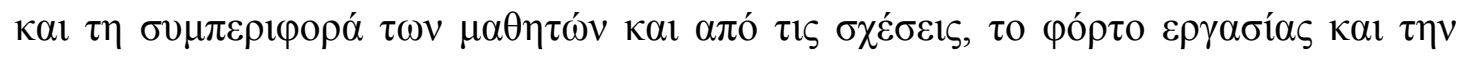

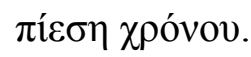

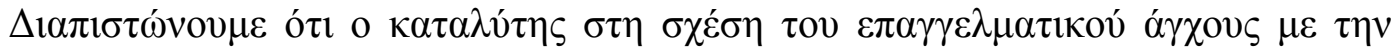

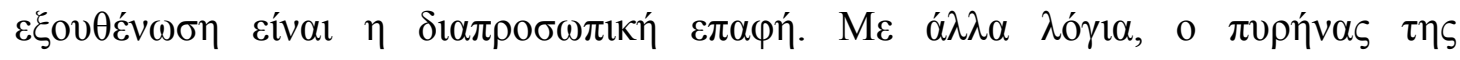

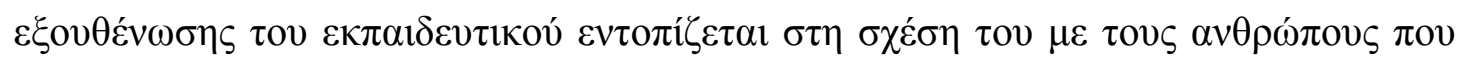

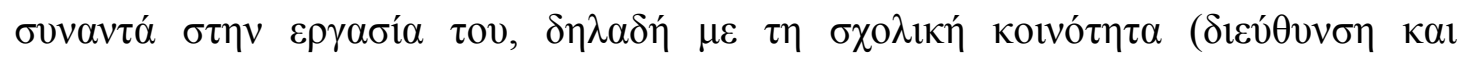

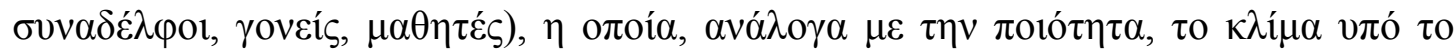




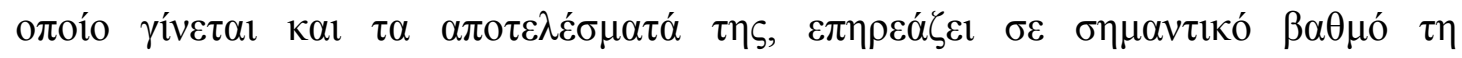

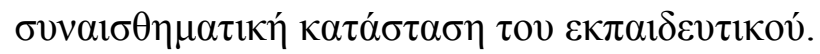

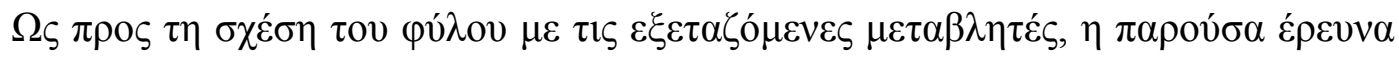

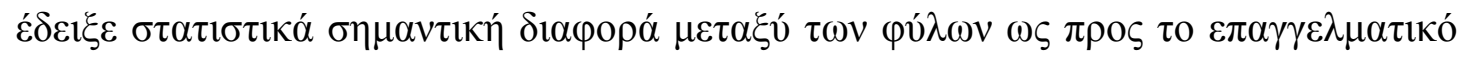

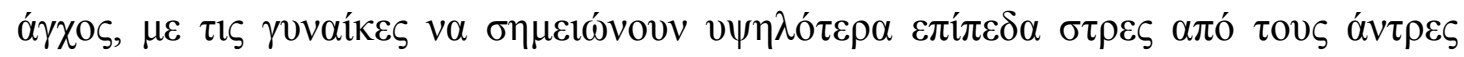

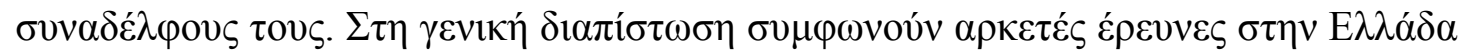

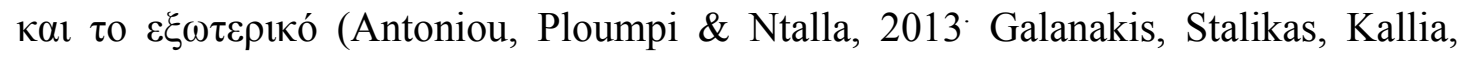

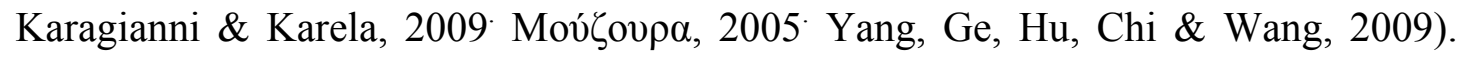

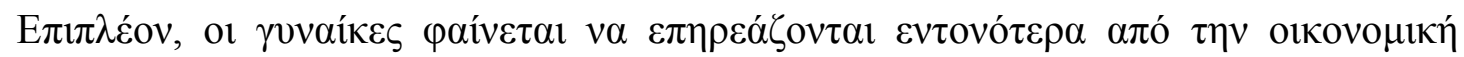

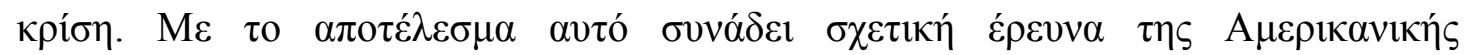

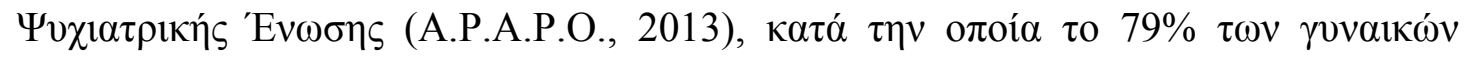

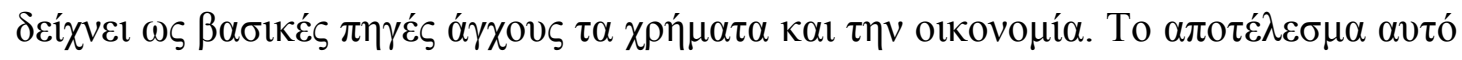

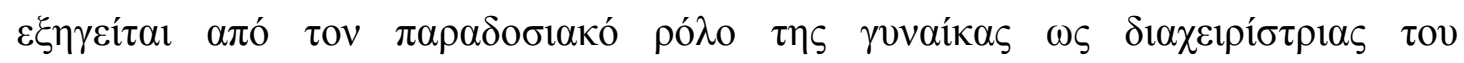

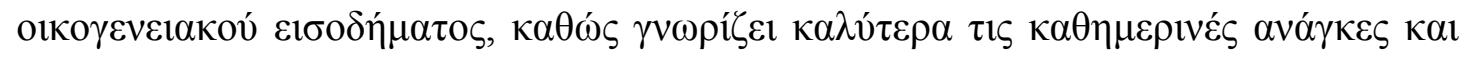

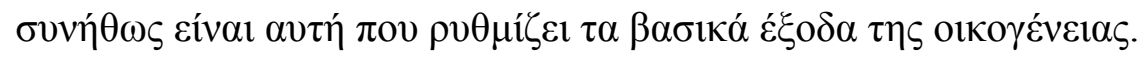

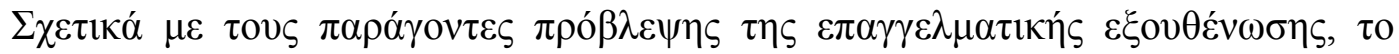

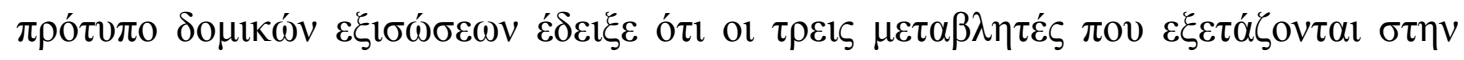

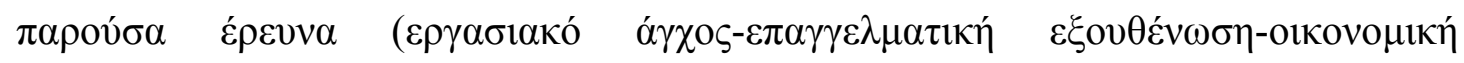

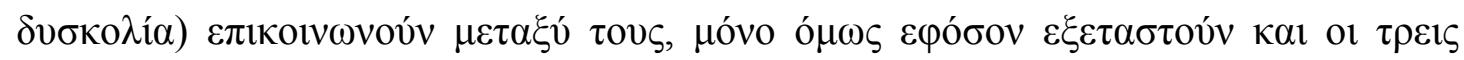

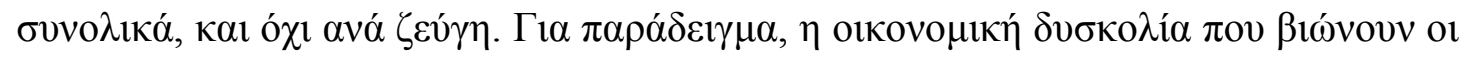

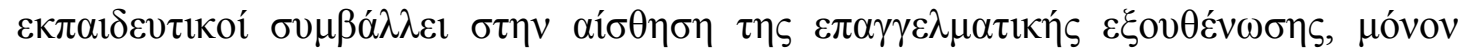

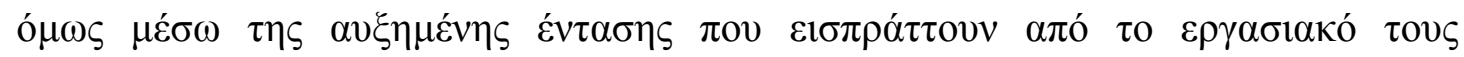

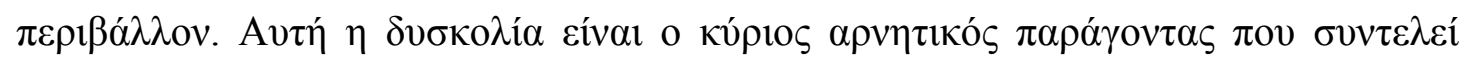

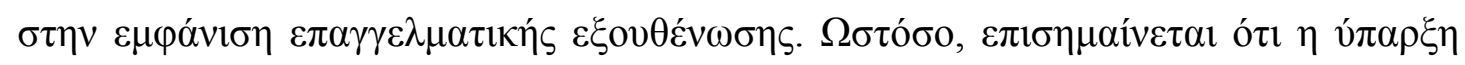

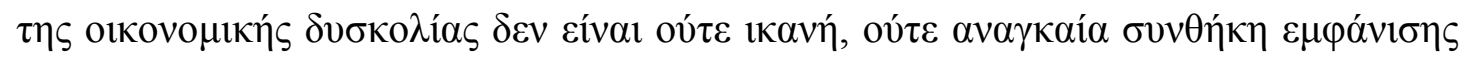


$\varepsilon \pi \alpha \gamma \gamma \varepsilon \lambda \mu \alpha \tau 1 \kappa \eta ́ \varsigma ~ \varepsilon \xi o v \theta \varepsilon ́ v \omega \sigma \eta \varsigma$.

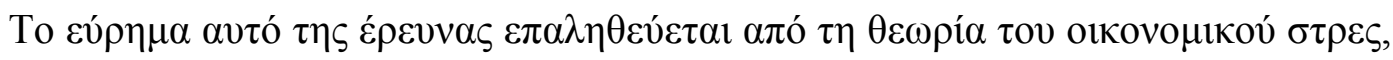

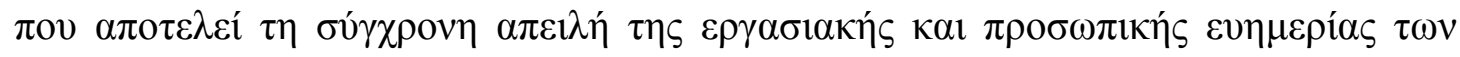

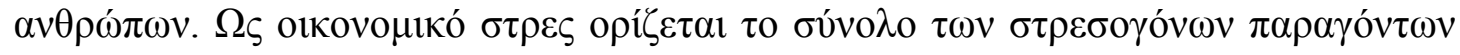

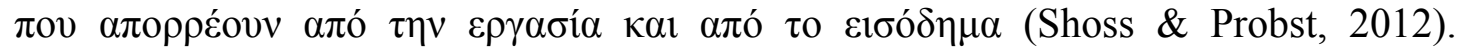

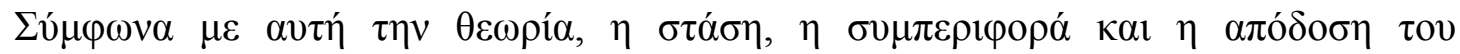

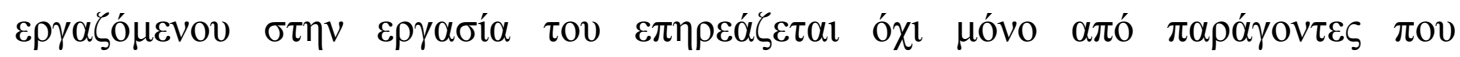

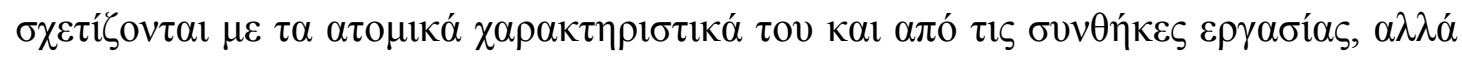

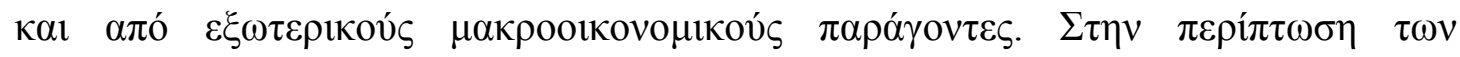

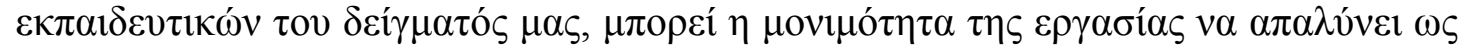

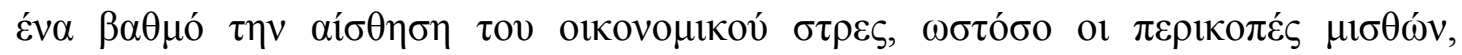

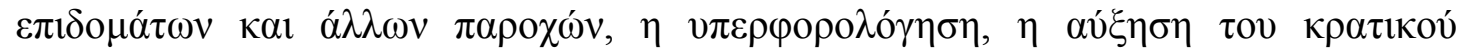

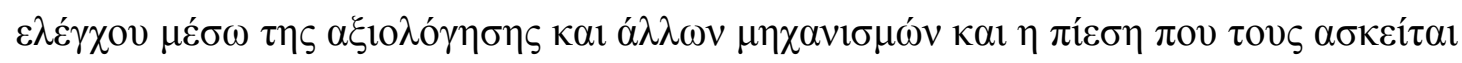

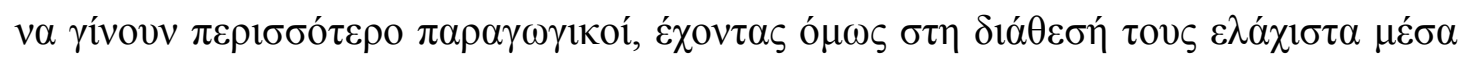

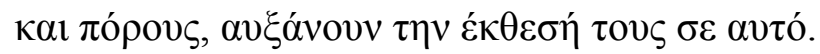

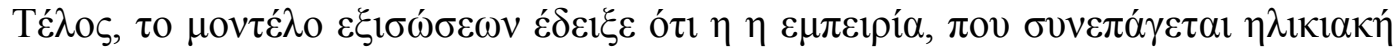

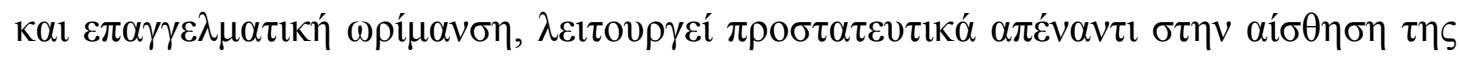

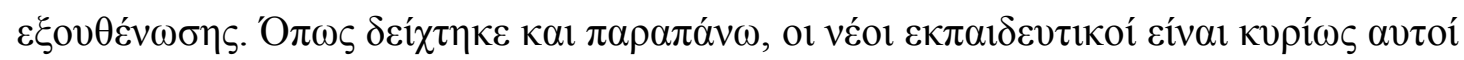

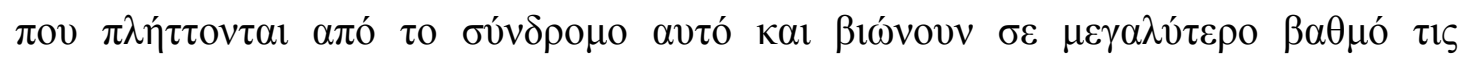

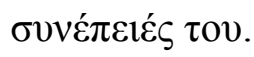




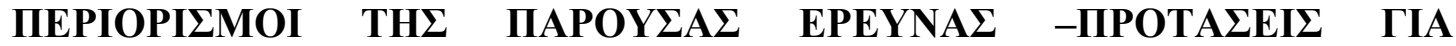

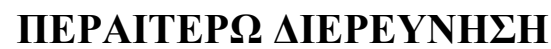

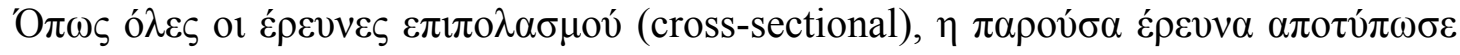

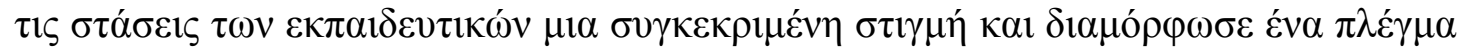

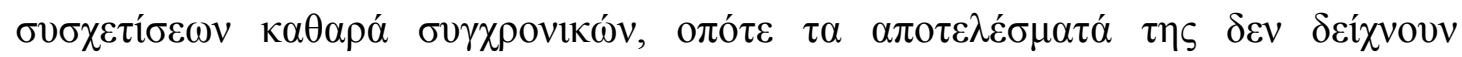

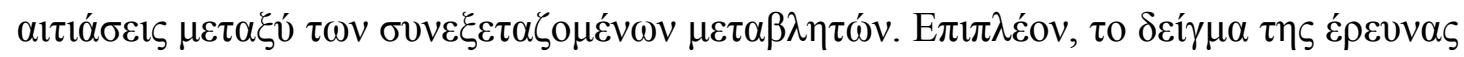

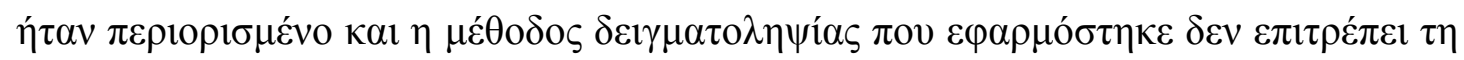

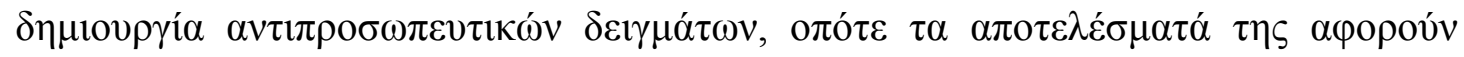

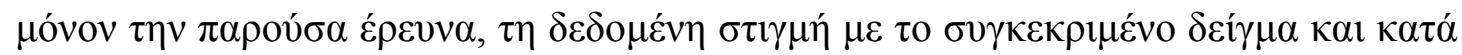

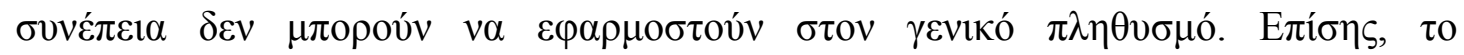

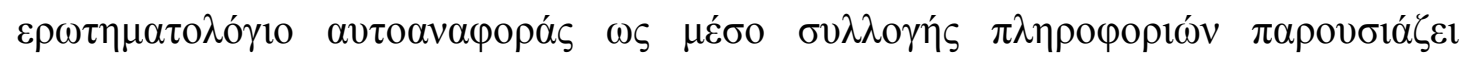

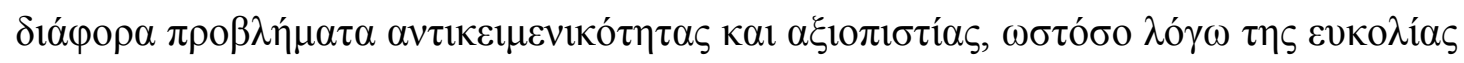

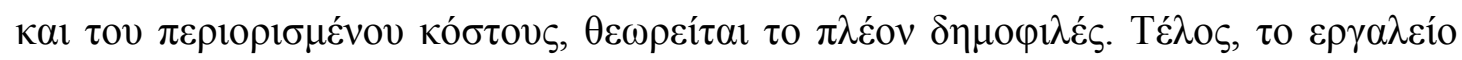

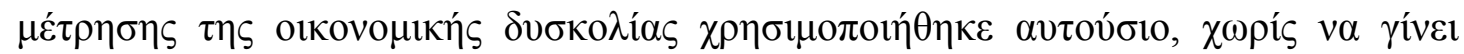

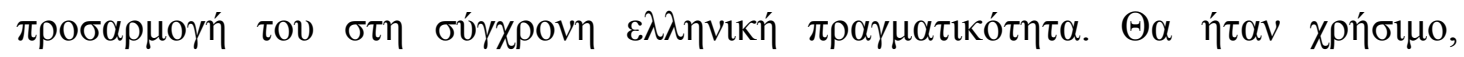

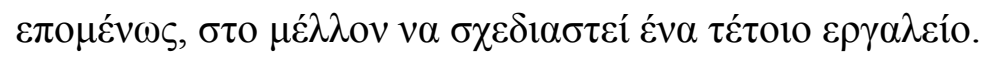

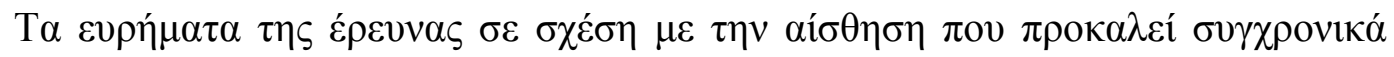

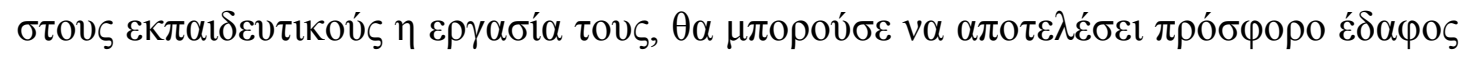

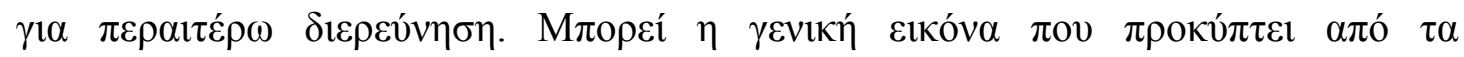

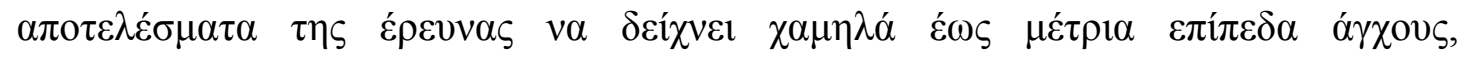

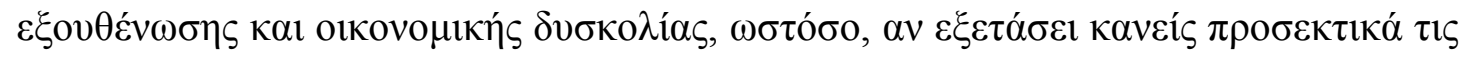

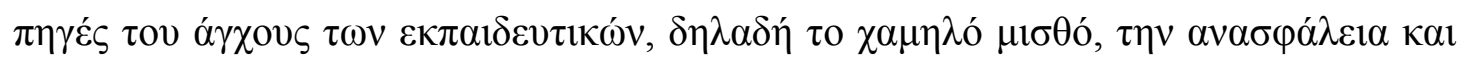

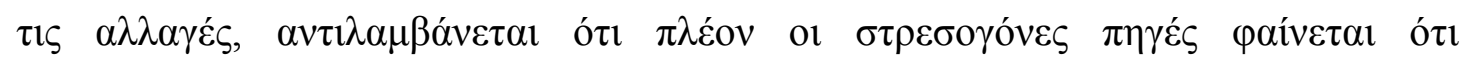

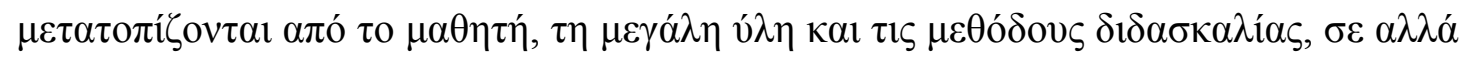




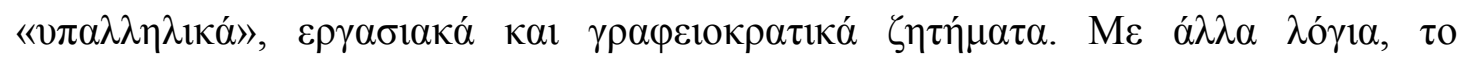

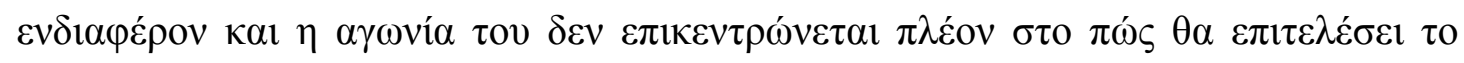

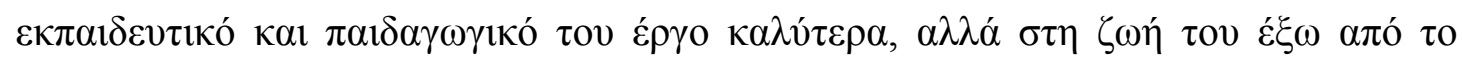

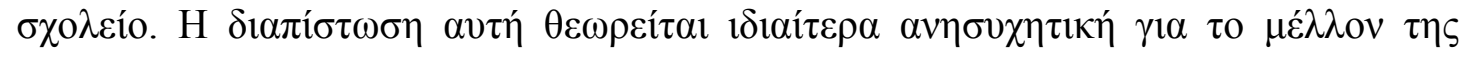

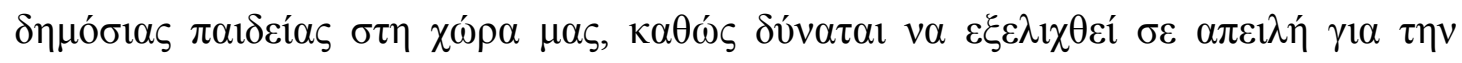

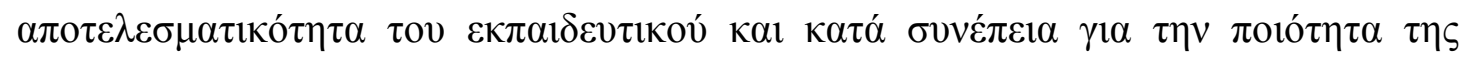

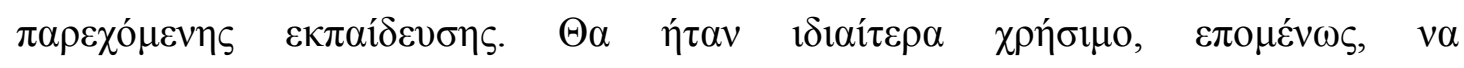

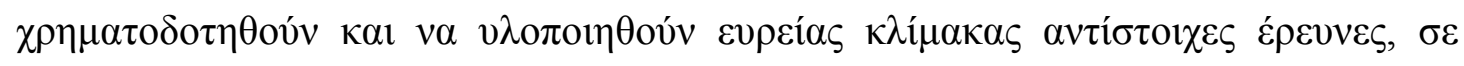

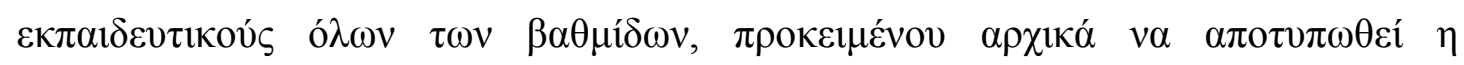

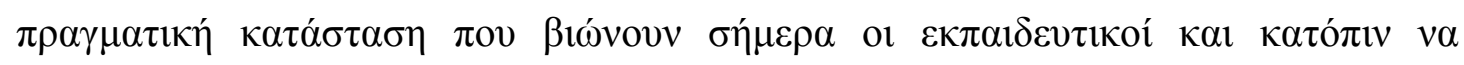

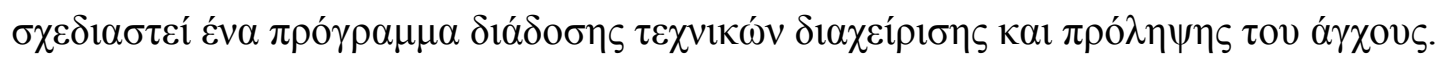




\section{ВIBАIОГРАФІА}

American Psychological Association Practice Organization (2013). APA Survey Finds US Employers Unresponsive to Employee Needs (www.apa.org).

Antoniou, A.S., Ploumpi, A. \& Ntalla, M. (2013). Occupational stress and professional burnout in teachers of primary and secondary education: the role of coping strategies. Psychology, 4 (3A), 349-355.

Barrera, M., Caples, H. \& Tein J-Y. (2001). The psychological sense of economic hardship: measurement models, validity and cross-ethnic equivalence for urban families. American journal of community psychology, 29(3), 493-517.

Borg, M. \& Riding, R. (1991). Occupational stress and satisfaction in teaching. British educational research journal, 17(3), 263-281.

Bullough, R. V., \& Draper, R. J. (2004). Mentoring and the emotions. Journal of education for teaching, 30, 271-288.

Calderhead, J. (2001). Handbook of research on teaching. In V. Richardson (Ed.), International experiences of teaching reform (4th ed.). Washington: American Educational Research Association.

Chang, M.-L. (2009). An appraisal perspective of teacher burnout: examining the emotional work of teachers. Educational psychology review, 21, 193-218.

Eurofound (2014), Third European Quality of Life Survey - Quality of life in Europe: Families in the economic crisis. Publications Office of the European Union, Luxembourg.

European Agency for Safety and Health at Work (2008). E-fact 31: Prevention of work-related stress in the education sector (https://osha.europa.eu).

European Agency for Safety and Health at Work (2009). OSH in figures: stress at work-facts and figures (https://osha.europa.eu). 
European Trade Union Committee for Education (E.T.U.C.E.) (2008). Report on the E.T.U.C.E. survey on teacher's work-related stress (http://etuce.homestead.com).

European Trade Union Committee for Education (E.T.U.C.E.), (2013). Survey - the continued impact of the crisis on teachers in Europe. Brussels. (http://etuce.homestead.com).

Galanakis, M., Stalikas, A., Kallia, H., Karagianni, C. \& Karela, C. (2009). Gender differences in experiencing occupational stress: the role of age, education and marital status. Stress and health, 25, 397-404.

Ganster, D. \& Rosen, C. (2013). Work stress and employee health: a multidisciplinary review. Journal of management, 39(5), 1085-1122.

Hargreaves, A. (2005). Educational change takes ages: Life, career and generational factors in teachers'emotional responses to educational change. Teaching and Teacher Education, 21(8), 967-983.

Hastings, R.P., Horne, S. \& Mitchell, G. (2004). Burnout in direct care staff in intellectual disability services: A factor analytic study of the Maslach Burnout Inventory. Journal of intellectual disability research, 48, 268-273.

Health and Safety Executive (2007). HSC Chair highlights real health and safety issues affecting Britain's workforce (http://www.hse.gov.uk).

Health and Safety Executive (2013). Stress-related and psychological disorders in Great Britain (www.hse.gov.uk).

Hossain, N., Byrne, B., Campbell, A., Harrison, E., McKinley, B. \& Shah, P. (2011). The impact of the global economic downturn on communities and poverty in the UK. Joseph Rowntree Foundation. 


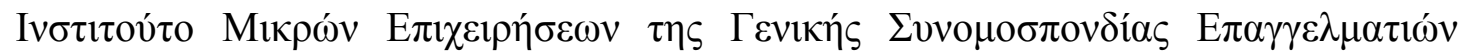

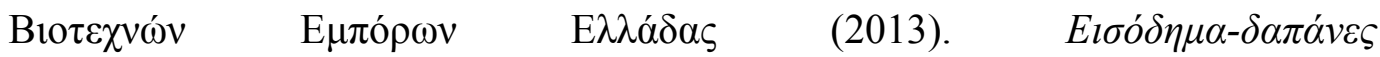

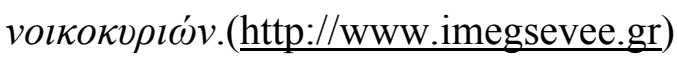

Jepson, E., \& Forrest, S. (2006). Individual contributory factors in teacher stress: The role of achievement striving and occupational commitment. The British journal of educational psychology, 76, 183-197.

Johnson, S., Cooper, C., Cartwright, S., Donald, I., Taylor, P.J. \& Millet, C. (2005). The experience of work-related stress across occupations. Journal of managerial psychology, 20(2), 178-187.

Kalyva, E. (2013). Stress in Greek primary school teachers working under conditions of financial crisis. Europe's journal of psychology, 9(1), 104-112 (doi:10.5964/ejop.v9i1.488).

Kondilis, E., Giannakopoulos, S., Gavana, M., Ierodiakonou, I., Waitzkin, E., \& Benos, A. (2013). Economic crisis, restrictive policies and the population's health and health care: the Greek case. American journal of public health, 103(6), 973979.

Kyriacou, C. (2001). Teacher stress: Directions of future research. Educational review, 53, 27-35.

Kyriacou, C. \& Sutcliffe, J. (1977). Teacher stress: a review. Educational review, 29(4), 299-306.

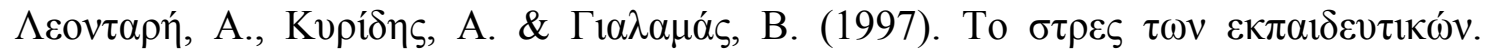

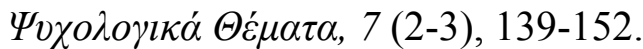

Maslach, C. \& Jackson, S.E. (1981). The measurement of experienced burnout. Journal of occupational behavior, 2, 99-113.

Maslach, C., Jackson, S. E., \& Leiter, M. P. (1996). The Maslach Burnout Inventory 
(3rd ed.). Palo Alto: Consulting Psychologists Press.

Maslach, C., Jackson, S.E. \& Schwab, R.L. (1996). MBI-Educators Survey (MBIES). In C.Maslach, S.E. Jackson \& R.L. Schwab (Ed.). MBI Manual. Palo Alto: Consulting Psychologists Press.

Maslach, C., Schaufeli, W.B., \& Leiter, M.P. (2001). Job burnout. In S.T. Fiske, D.L. Schacter, \& C. Zahn-Waxler (Ed.), Annual review of psychology, 52 (pp. 397422).

Mitrakos, T. (2014). Inequality, poverty and social welfare in Greece: distributional effects of austerity (working paper). $\Delta \mathrm{\iota} \alpha \dot{\varepsilon} \sigma \mu \mathrm{o}$ : http://www.bankofgreece.gr/BogEkdoseis/Paper2014174.pdf

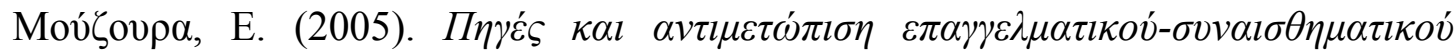

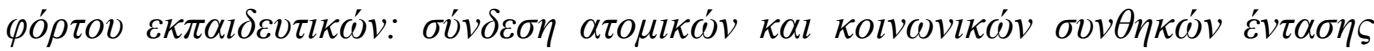

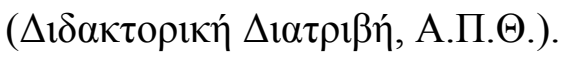

O.E.C.D. (Organisation for Economic Co-operation and Development), (2013a). Education at a Glance 2013: Highlights. $\Delta 1 \alpha \theta \dot{\varepsilon} \sigma \mu \mathrm{o} \sigma \tau$ :

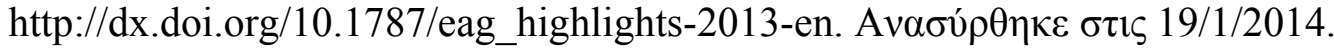

O.E.C.D. (Organisation for Economic Co-operation and Development), (2013b). “Teachers' salaries", in Government at a glance 2013. $\Delta \mathrm{x} \alpha \dot{\varepsilon} \sigma 1 \mu \mathrm{o}$ $\sigma \tau$ o:http://dx.doi.org/10.1787/gov_glance-2013-39-en.

Platsidou, M. \& Agaliotis, I. (2008). Burnout, job satisfaction and instructional assignment-related sources of stress in Greek special education teachers. International journal of disability, development and education, 55(1), 61-76.

Shoss, M.K. \& Probst, T.M. (2012). Multilevel outcomes of economic stress: an agenda for future research. In P.L. Perrewé, J.R.B. Halbesleben, C.C. Rosen (Ed.)The role of the economic crisis on occupational stress and well-being, 10. 
Emerald Group Publishing Ltd.

Steinhardt, M., Smith-Jaggars, S., Faulk, K. \& Gloria, C. (2011). Chronic work stress and depressive symptoms: assessing the mediating role of teacher burnout. Stress and health, 27, 420-429.

Travers, C.J. \& Cooper, C.L. (1993). Mental health, job satisfaction and occupational stress among UK teachers. Work and stress, 7, 203-219.

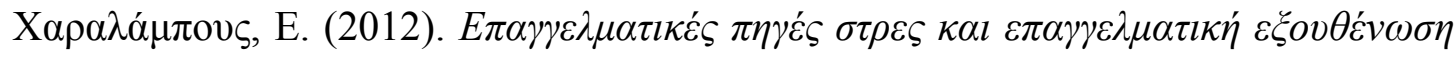

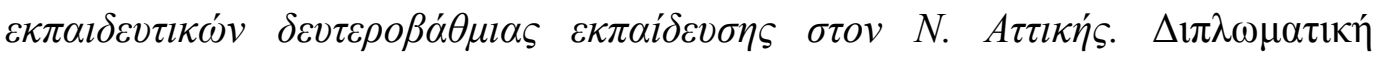

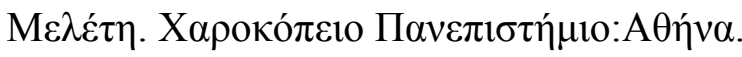

Yang, X., Ge, C., Hu, B., Chi, T. \& Wang, L. (2009). Relationship between quality of life and occupational stress among teachers. Public health, 123, 750-755.

Zembylas, M., \& Barker, H. (2007). Teachers' spaces for coping with change in the context of a reform effort. Journal of educational change, 8(3), 235-256. 